\title{
Chemical Structures and Biological Activities of Limonoids from the Genus Swietenia (Meliaceae)
}

\author{
Yun-Peng Sun ${ }^{1}$, Wen-Fang Jin ${ }^{1}$, Yong-Yue Wang ${ }^{1}$, Gang Wang ${ }^{1,2}$, Susan L. Morris-Natschke ${ }^{3}$, \\ Jin-Song Liu ${ }^{1,2}$, Guo-Kai Wang ${ }^{1,2,3, *}$ and Kuo-Hsiung Lee ${ }^{3,4, * \text { (iD }}$ \\ 1 School of Pharmacy, Anhui University of Chinese Medicine, Hefei 230012, China; \\ syp1good@163.com (Y.-P.S.); jwfmiji@163.com (W.-F.J.); wyy_1996@126.com (Y.-Y.W.); \\ kunhong_8@163.com (G.W.); jinsongliu@ahtcm.edu.cn (J.-S.L.) \\ 2 Synergetic Innovation Center of Anhui Authentic Chinese Medicine Quality Improvement, \\ Hefei 230012, China \\ 3 Natural Products Research Laboratories, Eshelman School of Pharmacy, University of North Carolina, \\ Chapel Hill, NC 27599-7568, USA; susan_natschke@unc.edu \\ 4 Chinese Medicine Research and Development Center, China Medical University and Hospital, \\ Taichung 40402, Taiwan \\ * Correspondences: wanggk@ahtcm.edu.cn (G.-K.W.); khlee@unc.edu (K.-H.L.); \\ Tel.: +86-551-68129167 (G.-K.W.); +1-919-9620066 (K.-H.L.)
}

Received: 29 May 2018; Accepted: 26 June 2018; Published: 29 June 2018

\begin{abstract}
Swietenia is a genus in the plant family Meliaceae. This genus contains seven to eight known species, found in the tropical and subtropical regions of the Americas and West Africa. Thus far, more than 160 limonoids have been isolated from four species of the genus Swietenia. Limonoids are rich in structure type and biological activity, and these compounds are the main active components in the Swietenia species. This paper will give a comprehensive overview of the recent phytochemical and pharmacological research on the terpenes from Swietenia plants and encourage further drug discovery research.
\end{abstract}

Keywords: genus Swietenia; limonoids; chemical components; biological activities

\section{Introduction}

The genus Swietenia (Meliaceae) includes 7-8 species, which are mainly distributed in the tropical and subtropical regions of the Americas. These plants have gradually been introduced and cultivated in Indonesia, Vietnam, and Yunnan, Gansu, and other regions of China, and have grown well. The species Swietenia mahagoni J. acq, Swietenia macrophylla King and Swietenia humilis Zucc are timber species widely used in traditional medicine. In addition, Swietenia aubrevilleana, a hybrid of $S$. mahagoni and S. macrophylla, has been increasingly studied [1]. Prior reports have validated some of the traditional uses or found promising bioactivities in the laboratory, such as antidiabetic [2-4], antimicrobial [5-7], antioxidant [8,9], anti-inflammatory [10], antitumor [11,12], and acaricidal properties [13]. Previous phytochemical studies revealed that various types of limonoids have been isolated from Swietenia species, along with smaller amounts of steroids, coumarins, phytosterols [14], lignans [15], polyphenols [16], and essential oils [17] also found.

Limonoids, classified as tetranortriterpenoids, are formed by the loss of four terminal carbons from a side chain on an apotirucallane or apoeuphane skeleton with cyclization to form a $17 \beta$-furan ring [18]. Limonoids are found mainly as important secondary metabolites in the fruits of Rutaceae and Meliaceae plants. Their extensive biological effects, such as anti-malarial [19], antifeedant [20], insecticidal [21], and antitumor properties [22], have attracted the attention of many researchers. 
Structurally, most limonoids found in the genus Swietenia are classified as mexicanolide- and phragmalin-type, but the structural types are abundant and varied.

\section{Chemical Components}

\subsection{Azadirone-Type and Evodulon-Type Limonoids}

Azadirone-type limonoids are characterized by a 3-oxo- $\Delta^{1,2}$ pattern and C-7 oxygenation. Mahonin (1) was first isolated from the cotyledons of S. mahagoni in 1989 [23] and republished by the same author in 1990 [24,25]. The structures of swieteliacates A and B (2 and 3), which contain a lactone ring rather than the more common furan ring at C-17, were first reported in 2018 [26]. Swimacronoid A (4), an evodulon-type limonoid, was obtained from S. macrophylla in 2013 [27] (Figure 1).

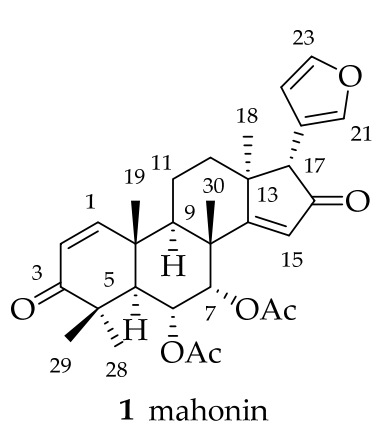

Figure 1. Chemical structures of azadirone-type and evodulon-type limonoids 1-4.

\subsection{Gedunin-Type Limonoids}

Gedunin-type limonoids with a $\delta$-lactone in ring $\mathrm{D}$ are derived from the azadirone class via a Baeyer-Villiger type ring expansion. 7-Deacetoxy-7-oxogedunin (5) was obtained from S. mahagoni, together with $6 \alpha$-acetoxygedunin (6) [24]. Compound 5 was also isolated from S. macrophylla [28] and S. aubrevilleana [1]. In 2009, compounds 8-13 were isolated from the fruits of S. mahagoni [29] (Figure 2, Table 1).

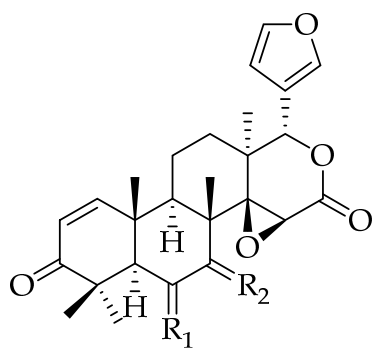

5-7

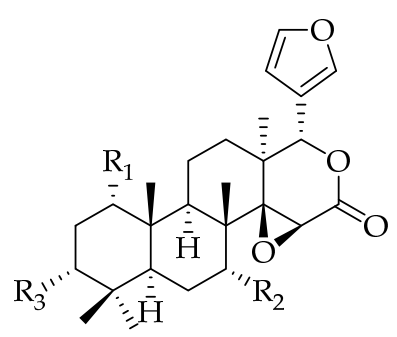

8-13

Figure 2. Chemical structures of gedunin-type limonoids 5-13.

Table 1. Structures and sources of gedunin-type limonoids 5-13.

\begin{tabular}{cccc}
\hline No. & Compounds & Substitution Groups & Sources \\
\hline 5 & 7-deacetoxy-7-oxogedunin & $\mathrm{R}_{1}=\mathrm{H}_{2}, \mathrm{R}_{2}=\mathrm{O}$ & $\begin{array}{c}\text { S. mahagoni }[24,29-31] \\
\text { S. macrophylla }[1,28,32], \\
\text { S. aubrevilleana }[1]\end{array}$ \\
\hline $\mathbf{6}$ & $6 \alpha$-acetoxygedunin & $\mathrm{R}_{1}=\mathrm{R}_{2}=\beta-\mathrm{H}, \alpha$-OAc & S. mahagoni [24], \\
\hline
\end{tabular}


Table 1. Cont.

\begin{tabular}{cccc}
\hline No. & Compounds & Substitution Groups & Sources \\
\hline $\mathbf{7}$ & $\begin{array}{c}\text { 7-deacetoxy-7 } \alpha- \\
\text { hydroxygedunin(deacetylgedunin) }\end{array}$ & $\mathrm{R}_{1}=\mathrm{H}_{2}, \mathrm{R}_{2}=\beta-\mathrm{H}, \alpha-\mathrm{OH}$ & $\begin{array}{l}\text { S. macrophylla [28], } \\
\text { S. aubrevilleana [1] }\end{array}$ \\
\hline $\mathbf{8}$ & 3-deacetylkhivorin & $\mathrm{R}_{1}=\mathrm{OAc}, \mathrm{R}_{2}=\mathrm{OAc}, \mathrm{R}_{3}=\mathrm{OH}$ & S. mahagoni [29] \\
\hline $\mathbf{9}$ & 3,7-dideacetylkhivorin & $\mathrm{R}_{1}=\mathrm{OAc}, \mathrm{R}_{2}=\mathrm{OH}, \mathrm{R}_{3}=\mathrm{OH}$ & S. mahagoni [29] \\
\hline $\mathbf{1 0}$ & 1,3,7-trideacetylkhivorin & $\mathrm{R}_{1}=\mathrm{OH}, \mathrm{R}_{2}=\mathrm{OH}, \mathrm{R}_{3}=\mathrm{OH}$ & S. mahagoni [29] \\
\hline $\mathbf{1 1}$ & khivorin & $\mathrm{R}_{1}=\mathrm{OAc}, \mathrm{R}_{2}=\mathrm{OAc}, \mathrm{R}_{3}=\mathrm{OAc}$ & S. mahagoni [29] \\
\hline $\mathbf{1 2}$ & 7-deacetylkhivorin & $\mathrm{R}_{1}=\mathrm{OAc}, \mathrm{R}_{2}=\mathrm{OH}, \mathrm{R}_{3}=\mathrm{OAc}$ & S. mahagoni [29] \\
\hline $\mathbf{1 3}$ & 1-deacetylkhivorin & $\mathrm{R}_{1}=\mathrm{OH}, \mathrm{R}_{2}=\mathrm{OAc}, \mathrm{R}_{3}=\mathrm{OAc}$ & S. mahagoni [29] \\
\hline
\end{tabular}

\subsection{Andirobin-Type Limonoids}

Andirobin-type limonoids are characterized by cleavages between C-7/8 and C-16/17 as well as the formation of a $\Delta 8,30$ exocyclic double bond and $\delta$-lactone $D$ ring. Secomahoganin (18) was first isolated from S. mahagoni in 1989 [23,24], and later from S. macrophylla in 2015 [33]. Multiple new andirobin-class limonoids, including deacetylsecomahoganin (19) [30], swiemahogin A (20) [34], and swietmanin J (21) [29], were obtained from S. macrophylla (Figure 3, Table 2).

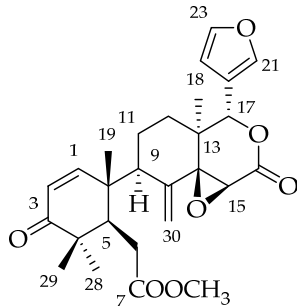

14

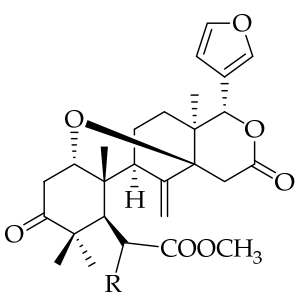

15-17

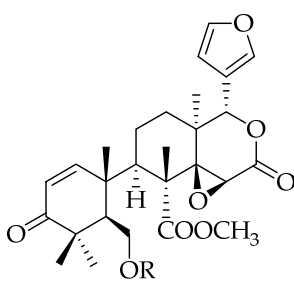

18-19

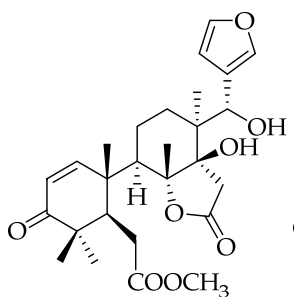

20

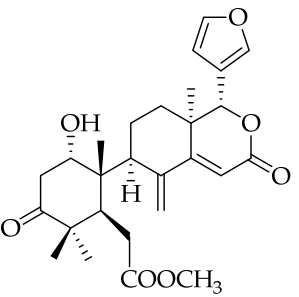

21

Figure 3. Chemical structures of andirobin-type limonoids 14-21.

Table 2. Structures and sources of andirobin-type limonoids 14-21.

\begin{tabular}{cccc}
\hline No. & Compound & Substitution Groups & Sources \\
\hline $\mathbf{1 4}$ & andirobin & S. macrophylla [1,35] \\
\hline $\mathbf{1 5}$ & methylangolensate & $\mathrm{R}=\mathrm{H}$ & $\begin{array}{c}\text { S. mahagoni [24,29,31,36], } \\
\text { S. macrophylla [27] }\end{array}$ \\
\hline $\mathbf{1 6}$ & $\begin{array}{c}\text { 6-hydroxy derivative (methyl 6- } \\
\text { hydroxyangolensate) }\end{array}$ & $\mathrm{R}=\mathrm{OH}$ & $\begin{array}{c}\text { S. mahagoni [29,30,36,37], } \\
\text { S. aubrevilleana [1], } \\
\text { S. macrophylla [27] }\end{array}$ \\
\hline $\mathbf{1 7}$ & 6-acetoxyangolensate & $\mathrm{R}=\mathrm{OAc}$ & S. macrophylla [27] \\
\hline $\mathbf{1 8}$ & secomahoganin & $\mathrm{R}=\mathrm{Ac}$ & $\begin{array}{c}\text { S. mahagoni [23-25], } \\
\text { S. macrophylla [33] }\end{array}$ \\
\hline $\mathbf{1 9}$ & deacetylsecomahoganin & $\mathrm{R}=\mathrm{H}$ & $\begin{array}{c}\text { S. mahagoni [30], } \\
\text { S. macrophylla [27] }\end{array}$ \\
\hline $\mathbf{2 0}$ & swiemahogin A & & S. mahagoni [34] \\
\hline $\mathbf{2 1}$ & swietmanin J & S. mahagoni [29] \\
\hline
\end{tabular}




\subsection{Mexicanolide-Type Limonoids}

A total of 77 mexicanolide-type limonoids, 22-98, have been reported from Swietenia species, and most were isolated from S. mahagoni and S. macrophylla. In a few reports, mexicanolide-type limonoids have also been found in S. humilis, for example, humilin B (88) [38], humilinolides A-H (89-90, 50-52, 91, 61, 98) [39-42], and 2-hydroxy-destigloyl-6-deoxyswietenine acetate (60) [42]. Swietenolide (23), 6-O-acetylswietenolide (25), and 3,6-O,O-diacetylswietenolide (27) were also obtained from S. aubrevilleana [1]. Other related interesting structures have been found in Swietenia species. Kadota et al. discovered a novel dimeric limonoid, mahagonin (77), from an oily fraction of the ether extract of $S$. mahagoni [43]. In addition, compound $\mathbf{7 8}$ was extracted from the seeds of S. macrophylla. The crystal structure contains 0.25 molecules of water and is stabilized by $\mathrm{O}-\mathrm{H} \cdots \mathrm{O}$ and weak $\mathrm{C}-\mathrm{H} \cdots$ O hydrogen bonds [44] (Figure 4, Table 3).
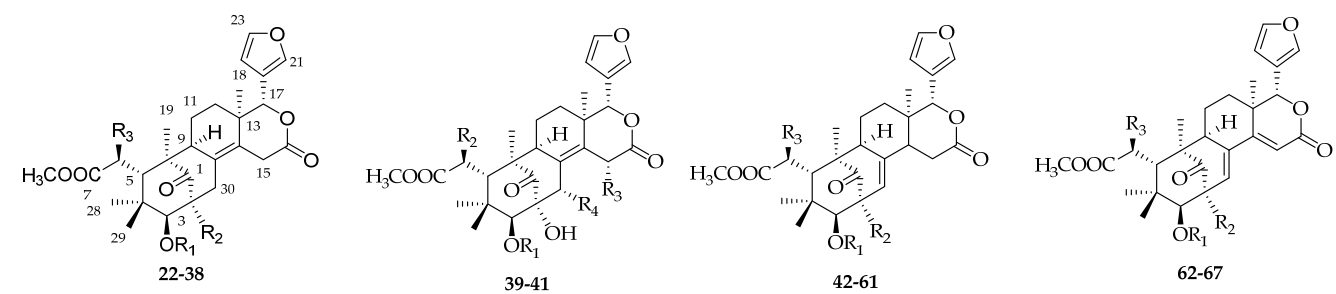

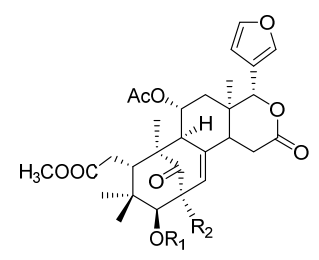

$68-71$

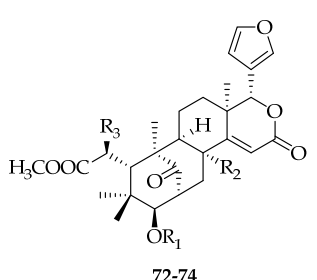

$72-74$

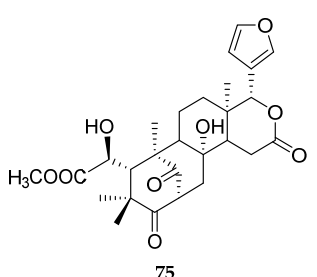

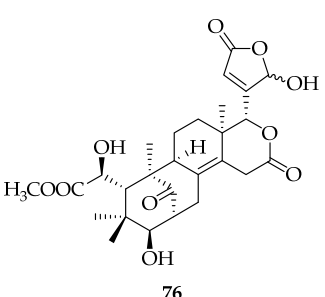

76
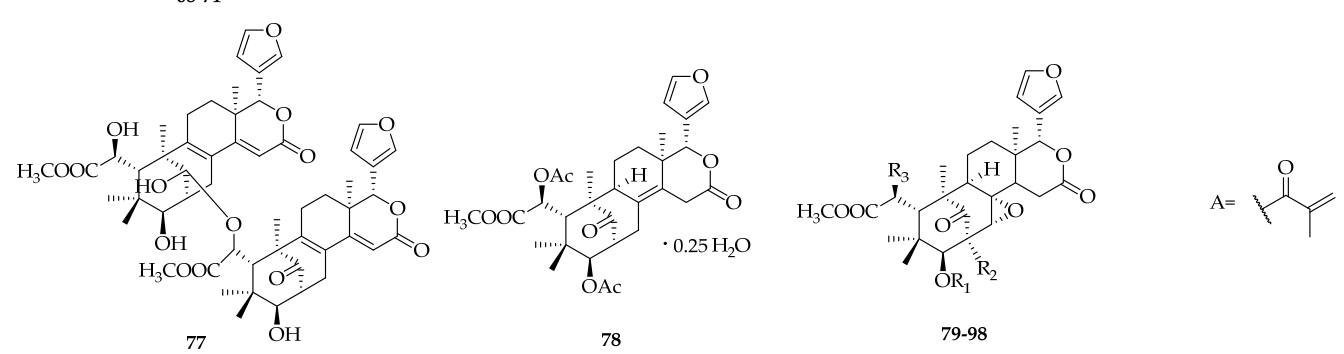

Figure 4. Chemical structures of mexicanolide-type limonoids 22-98.

Table 3. Structures and sources of mexicanolide-type limonoids 22-98.

\begin{tabular}{cccc}
\hline No. & Compounds & Substitution Groups & Sources \\
\hline $\mathbf{2 2}$ & mexicanolide & $\mathrm{R}_{1}=\mathrm{O}, \mathrm{R}_{2}=\mathrm{H}, \mathrm{R}_{3}=\mathrm{H}$ & S. mahagoni [29] \\
\hline $\mathbf{2 3}$ & swietenolide & $\mathrm{R}_{1}=\mathrm{H}, \mathrm{R}_{2}=\mathrm{H}, \mathrm{R}_{3}=\mathrm{OH}$ & $\begin{array}{c}\text { S. mahagoni }[24,45-47], \\
\text { S. aubrevilleana }[1], \\
\text { S. macrophylla } \\
{[1,32,48-50]}\end{array}$ \\
\hline $\mathbf{2 4}$ & 3-O-acetylswietenolide & $\mathrm{R}_{1}=\mathrm{Ac}, \mathrm{R}_{2}=\mathrm{H}, \mathrm{R}_{3}=\mathrm{OH}$ & $\begin{array}{c}\text { S. mahagoni }[24,46,51,52], \\
\text { S. macrophylla }[32,48]\end{array}$ \\
\hline $\mathbf{2 6}$ & 6-O-acetylswietenolide & $\mathrm{R}_{1}=\mathrm{H}, \mathrm{R}_{2}=\mathrm{H}, \mathrm{R}_{3}=\mathrm{OAc}$ & $\begin{array}{c}\text { S. mahagoni }[24,51], \\
\text { S. macrophylla }[1,48,53], \\
\text { S. aubrevilleana }[1]\end{array}$ \\
\hline 3-O-tigloyl-6-O-acetylswietenolide & $\mathrm{R}_{1}=\mathrm{Tig}, \mathrm{R}_{2}=\mathrm{H}, \mathrm{R}_{3}=\mathrm{OAc}$ & $\begin{array}{c}\text { S. mahagoni }[24,46], \\
\text { S. macrophylla }[14,32,48],\end{array}$ \\
\hline
\end{tabular}


Table 3. Cont.

\begin{tabular}{|c|c|c|c|}
\hline No. & Compounds & Substitution Groups & Sources \\
\hline 27 & 3,6-O,O-diacetylswietenolide & $\mathrm{R}_{1}=\mathrm{Ac}, \mathrm{R}_{2}=\mathrm{H}, \mathrm{R}_{3}=\mathrm{OAc}$ & $\begin{array}{c}\text { S. mahagoni }[24,46,51] \\
\text { S. macrophylla } \\
{[1,14,48,50,54]} \\
\text { S. aubrevilleana }[1]\end{array}$ \\
\hline 28 & 3-O-tigloylswietenolide & $\mathrm{R}_{1}=\mathrm{Tig}, \mathrm{R}_{2}=\mathrm{H}, \mathrm{R}_{3}=\mathrm{OH}$ & $\begin{array}{l}\text { S. mahagoni }[24,46] \text {, } \\
\text { S. macrophylla }[14,48,55] \text {, }\end{array}$ \\
\hline 29 & khayasin $\mathrm{T}$ & $\mathrm{R}_{1}=\mathrm{Tig}, \mathrm{R}_{2}=\mathrm{H}, \mathrm{R}_{3}=\mathrm{H}$ & $\begin{array}{l}\text { S. mahagoni }[24,29] \text {, } \\
\text { S. macrophylla }[1,14,48]\end{array}$ \\
\hline 30 & proceranolide & $\mathrm{R}_{1}=\mathrm{H}, \mathrm{R}_{2}=\mathrm{H}, \mathrm{R}_{3}=\mathrm{H}$ & $\begin{array}{l}\text { S. mahagoni }[24,48] \text {, } \\
\text { S. macrophylla }[32,33]\end{array}$ \\
\hline 31 & 2-hydroxy-3-O-tigloylswietenolide & $\mathrm{R}_{1}=\mathrm{Tig}, \mathrm{R}_{2}=\mathrm{OH}, \mathrm{R}_{3}=\mathrm{OH}$ & S. mahagoni $[30,47]$ \\
\hline 32 & 3-O-propionylproceranolide & $\mathrm{R}_{1}=\mathrm{COEt}, \mathrm{R}_{2}=\mathrm{H}, \mathrm{R}_{3}=\mathrm{H}$ & S. macrophylla [48] \\
\hline 33 & fissinolide & $\mathrm{R}_{1}=\mathrm{Ac}, \mathrm{R}_{2}=\mathrm{H}, \mathrm{R}_{3}=\mathrm{H}$ & $\begin{array}{l}\text { S. macrophylla }[32,33,48] \text {, } \\
\text { S. mahagoni }[29]\end{array}$ \\
\hline 34 & 2-hydroxy-3-O-isobutyrylproceranolide & $\mathrm{R}_{1}=\mathrm{iBu}, \mathrm{R}_{2}=\mathrm{OH}, \mathrm{R}_{3}=\mathrm{H}$ & S. mahagoni [29] \\
\hline 35 & 2-hydroxy-3-O-benzoylproceranolide & $\mathrm{R}_{1}=\mathrm{Bz}, \mathrm{R}_{2}=\mathrm{OH}, \mathrm{R}_{3}=\mathrm{H}$ & S. mahagoni [29] \\
\hline 36 & 2-hydroxyfissinolide & $\mathrm{R}_{1}=\mathrm{Ac}, \mathrm{R}_{2}=\mathrm{OH}, \mathrm{R}_{3}=\mathrm{H}$ & S. mahagoni [29] \\
\hline 37 & 2,3-dihydroxy-3-deoxymexicanolide & $\mathrm{R}_{1}=\mathrm{H}, \mathrm{R}_{2}=\mathrm{OH}, \mathrm{R}_{3}=\mathrm{H}$ & S. mahagoni [29] \\
\hline 38 & 2-hydroxy-6-deoxyswietenolide tiglate & $\mathrm{R}_{1}=\mathrm{Tig}, \mathrm{R}_{2}=\mathrm{OH}, \mathrm{R}_{3}=\mathrm{H}$ & S. mahagoni [29] \\
\hline 39 & augustineolide & $\mathrm{R}_{1}=\mathrm{Tig}, \mathrm{R}_{2}=\mathrm{OH}, \mathrm{R}_{3}=\mathrm{OAc}, \mathrm{R}_{4}=\mathrm{OiBu}$ & S. macrophylla [1] \\
\hline 40 & swietmanin E & $\mathrm{R}_{1}=\mathrm{Tig}, \mathrm{R}_{2}=\mathrm{H}, \mathrm{R}_{3}=\mathrm{OH}, \mathrm{R}_{4}=\mathrm{H}$ & S. mahagoni [29] \\
\hline 41 & swietmanin F & $\mathrm{R}_{1}=\mathrm{Ac}, \mathrm{R}_{2}=\mathrm{H}, \mathrm{R}_{3}=\mathrm{OH}, \mathrm{R}_{4}=\mathrm{H}$ & S. mahagoni [29] \\
\hline 42 & swietenine & $\mathrm{R}_{1}=\mathrm{Tig}, \mathrm{R}_{2}=\mathrm{H}, \mathrm{R}_{3}=\mathrm{OH}$ & $\begin{array}{c}\text { S. mahagoni }[24,35,45,46] \\
\text { S. macrophylla } \\
{[14,33,36,48,49,56,57]}\end{array}$ \\
\hline 43 & swietenine B & $\mathrm{R}_{1}=\mathrm{COEt}, \mathrm{R}_{2}=\mathrm{H}, \mathrm{R}_{3}=\mathrm{OH}$ & S. mahagoni [24] \\
\hline 44 & swietenine $\mathrm{C}$ & $\mathrm{R}_{1}=\mathrm{iBu}, \mathrm{R}_{2}=\mathrm{H}, \mathrm{R}_{3}=\mathrm{OH}$ & $\begin{array}{l}\text { S. mahagoni }[24,58], S . \\
\text { humilis }[41]\end{array}$ \\
\hline 45 & swietenine D & $\mathrm{R}_{1}=\mathrm{A}, \mathrm{R}_{2}=\mathrm{H}, \mathrm{R}_{3}=\mathrm{OH}$ & S. mahagoni [24] \\
\hline 46 & swietenine E & $\mathrm{R}_{1}=\mathrm{Piv}, \mathrm{R}_{2}=\mathrm{H}, \mathrm{R}_{3}=\mathrm{OH}$ & S. mahagoni [24] \\
\hline 47 & swietenine F & $\mathrm{R}_{1}=\mathrm{Bz}, \mathrm{R}_{2}=\mathrm{H}, \mathrm{R}_{3}=\mathrm{OH}$ & S. mahagoni [24] \\
\hline 48 & $\begin{array}{c}\text { swietenine acetate } \\
(6-O \text {-acetylswietenine })\end{array}$ & $\mathrm{R}_{1}=\mathrm{Tig}, \mathrm{R}_{2}=\mathrm{H}, \mathrm{R}_{3}=\mathrm{OAc}$ & $\begin{array}{l}\text { S. mahagoni }[24,46], \\
\text { S. macrophylla }[14,33,49]\end{array}$ \\
\hline 49 & 6-desoxyswietenine (febrifugin) & $\mathrm{R}_{1}=\mathrm{Tig}, \mathrm{R}_{2}=\mathrm{H}, \mathrm{R}_{3}=\mathrm{H}$ & $\begin{array}{l}\text { S. mahagoni }[46,59] \text {, } \\
\text { S. macrophylla }[1,14,48]\end{array}$ \\
\hline 50 & humilinolide C & $\mathrm{R}_{1}=\mathrm{Tig}, \mathrm{R}_{2}=\mathrm{OAc}, \mathrm{R}_{3}=\mathrm{H}$ & S. humilis [39-41] \\
\hline 51 & humilinolide D & $\mathrm{R}_{1}=\mathrm{Ac}, \mathrm{R}_{2}=\mathrm{OH}, \mathrm{R}_{3}=\mathrm{OAc}$ & S. humilis [39-41] \\
\hline 52 & $\begin{array}{c}\text { humilinolide E } \\
\text { (6-O-acetyl-2-hydroxyswietenin) }\end{array}$ & $\mathrm{R}_{1}=\mathrm{Tig}, \mathrm{R}_{2}=\mathrm{OH}, \mathrm{R}_{3}=\mathrm{OAc}$ & $\begin{array}{l}\text { S. humilis }[41], \\
\text { S. mahagoni }[31,57]\end{array}$ \\
\hline 53 & $\begin{array}{l}\text { methyl-2-hydroxy-3-b-isobutyroxy- } \\
\text { 1-oxomeliac-8(30)-enate }\end{array}$ & $\mathrm{R}_{1}=\mathrm{iBu}, \mathrm{R}_{2}=\mathrm{OH}, \mathrm{R}_{3}=\mathrm{H}$ & S. humilis $[38,41]$ \\
\hline 54 & $\begin{array}{l}\text { methyl-2-hydroxy-3-b-tigloyloxy- } \\
\text { 1-oxomeliac-8(30)-enate }\end{array}$ & $\mathrm{R}_{1}=\mathrm{Tig}, \mathrm{R}_{2}=\mathrm{OH}, \mathrm{R}_{3}=\mathrm{H}$ & $\begin{array}{l}\text { S. humilis [41], } \\
\text { S. macrophylla [58], } \\
\text { S. mahagoni [31] }\end{array}$ \\
\hline 55 & 2-hydroxyswietenine & $\mathrm{R}_{1}=\mathrm{Tig}, \mathrm{R}_{2}=\mathrm{OH}, \mathrm{R}_{3}=\mathrm{OH}$ & $\begin{array}{l}\text { S. mahagoni }[31,36,56] \text {, } \\
\text { S. macrophylla }[1,58]\end{array}$ \\
\hline 56 & 6-acetoxyhumilinolide $\mathrm{C}$ & $\mathrm{R}_{1}=\mathrm{Tig}, \mathrm{R}_{2}=\mathrm{OAc}, \mathrm{R}_{3}=\mathrm{OAc}$ & S. aubrevilleana [1] \\
\hline 57 & granatumin $\mathrm{H}$ & $\mathrm{R}_{1}=\mathrm{iBu}, \mathrm{R}_{2}=\mathrm{H}, \mathrm{R}_{3}=\mathrm{H}$ & S. macrophylla [48] \\
\hline 58 & swieteliacate C & $\mathrm{R}_{1}=\mathrm{COEt}, \mathrm{R}_{2}=\mathrm{H}, \mathrm{R}_{3}=\mathrm{H}$ & S. macrophylla [26] \\
\hline 59 & 6-O-acetylswietenin B & $\mathrm{R}_{1}=\mathrm{COEt}, \mathrm{R}_{2}=\mathrm{H}, \mathrm{R}_{3}=\mathrm{OAc}$ & S. macrophylla [48] \\
\hline 60 & $\begin{array}{l}\text { 2-hydroxy-destigloyl-6-deoxyswietenine } \\
\text { acetate }\end{array}$ & $\mathrm{R}_{1}=\mathrm{Ac}, \mathrm{R}_{2}=\mathrm{OH}, \mathrm{R}_{3}=\mathrm{H}$ & S. humilis [42] \\
\hline
\end{tabular}


Table 3. Cont.

\begin{tabular}{|c|c|c|c|}
\hline No. & Compounds & Substitution Groups & Sources \\
\hline 61 & humilinolide G & $\mathrm{R}_{1}=\mathrm{iBu}, \mathrm{R}_{2}=\mathrm{OAc}, \mathrm{R}_{3}=\mathrm{H}$ & S. humilis [42] \\
\hline 62 & swielimonoid A & $\mathrm{R}_{1}=\mathrm{Tig}, \mathrm{R}_{2}=\mathrm{H}, \mathrm{R}_{3}=\mathrm{OH}$ & S. macrophylla [60] \\
\hline 63 & swielimonoid B & $\mathrm{R}_{1}=\mathrm{COEt}, \mathrm{R}_{2}=\mathrm{H}, \mathrm{R}_{3}=\mathrm{OH}$ & S. macrophylla [60] \\
\hline 64 & swietmanin G & $\mathrm{R}_{1}=\mathrm{iBu}, \mathrm{R}_{2}=\mathrm{OH}, \mathrm{R}_{3}=\mathrm{H}$ & S. mahagoni [29] \\
\hline 65 & swietmanin $\mathrm{H}$ & $\mathrm{R}_{1}=\mathrm{Ac}, \mathrm{R}_{2}=\mathrm{OH}, \mathrm{R}_{3}=\mathrm{H}$ & S. mahagoni [29] \\
\hline 66 & swietmanin I & $\mathrm{R}_{1}=\mathrm{Tig}, \mathrm{R}_{2}=\mathrm{OH}, \mathrm{R}_{3}=\mathrm{H}$ & S. mahagoni [29] \\
\hline 67 & seneganolide A & $\mathrm{R}_{1}=\mathrm{H}, \mathrm{R}_{2}=\mathrm{H}, \mathrm{R}_{3}=\mathrm{H}$ & S. mahagoni [29] \\
\hline 68 & swietmanin A & $\mathrm{R}_{1}=\mathrm{iBu}, \mathrm{R}_{2}=\mathrm{H}$ & S. mahagoni [29] \\
\hline 69 & swietmanin B & $\mathrm{R}_{1}=\mathrm{Ac}, \mathrm{R}_{2}=\mathrm{H}$ & S. mahagoni [29] \\
\hline 70 & swietmanin C & $\mathrm{R}_{1}=\mathrm{H}, \mathrm{R}_{2}=\mathrm{H}$ & S. mahagoni [29] \\
\hline 71 & swietmanin D & $\mathrm{R}_{1}=\mathrm{Ac}, \mathrm{R}_{2}=\mathrm{OAc}$ & S. mahagoni [29] \\
\hline 72 & $8 \alpha$-hydroxycarapin & $\mathrm{R}_{1}=\mathrm{O}, \mathrm{R}_{2}=\mathrm{OH}, \mathrm{R}_{3}=\mathrm{H}$ & S. mahagoni [29] \\
\hline 73 & $3 \beta, 6$-dihydroxydihydrocarapin & $\mathrm{R}_{1}=\mathrm{H}, \mathrm{R}_{2}=\mathrm{H}, \mathrm{R}_{3}=\mathrm{OH}$ & $\begin{array}{l}\text { S. macrophylla [1], } \\
\text { S. aubrevilleana [1] }\end{array}$ \\
\hline 74 & swieteliacate E & $\mathrm{R}_{1}=\mathrm{H}, \mathrm{R}_{2}=\mathrm{OH}, \mathrm{R}_{3}=\mathrm{OH}$ & S. macrophylla [26] \\
\hline 75 & khayanone & & S. macrophylla [37] \\
\hline 76 & swieteliacate D & & S. macrophylla [26] \\
\hline 77 & mahagonin & & $\begin{array}{l}\text { S. mahagoni [43], } \\
\text { S. macrophylla [26] }\end{array}$ \\
\hline 78 & $\begin{array}{c}\text { 3,6-di-O-acetylswietenolide } \\
\text { 0.25-hydrate }\end{array}$ & & S. macrophylla [44] \\
\hline 79 & swietemahonin A & $\mathrm{R}_{1}=\mathrm{COEt}, \mathrm{R}_{2}=\mathrm{H}, \mathrm{R}_{3}=\mathrm{OH}$ & S. mahagoni $[24,45,51,52]$ \\
\hline 80 & swietemahonin B & $\mathrm{R}_{1}=\mathrm{COEt}, \mathrm{R}_{2}=\mathrm{H}, \mathrm{R}_{3}=\mathrm{OAc}$ & $\begin{array}{l}\text { S. mahagoni }[24,45] \text {, } \\
\text { S. macrophylla }[48]\end{array}$ \\
\hline 81 & swietemahonin C & $\mathrm{R}_{1}=\mathrm{iBu}, \mathrm{R}_{2}=\mathrm{H}, \mathrm{R}_{3}=\mathrm{OAc}$ & S. mahagoni $[24,41,45]$ \\
\hline 82 & swietemahonin D & $\mathrm{R}_{1}=\mathrm{Ac}, \mathrm{R}_{2}=\mathrm{H}, \mathrm{R}_{3}=\mathrm{OH}$ & S. mahagoni $[24,45,51]$ \\
\hline 83 & swietemahonin E & $\mathrm{R}_{1}=\mathrm{Tig}, \mathrm{R}_{2}=\mathrm{H}, \mathrm{R}_{3}=\mathrm{OH}$ & $\begin{array}{l}\text { S. mahagoni }[24,45,51,52] \text {, } \\
\text { S. macrophylla }[1,14,33,48]\end{array}$ \\
\hline 84 & swietemahonin F & $\mathrm{R}_{1}=\mathrm{Tig}, \mathrm{R}_{2}=\mathrm{H}, \mathrm{R}_{3}=\mathrm{OAc}$ & $\begin{array}{l}\text { S. mahagoni }[24,45] \text {, } \\
\text { S. macrophylla }[1,32,33]\end{array}$ \\
\hline 85 & swietemahonin G & $\mathrm{R}_{1}=\mathrm{Tig}, \mathrm{R}_{2}=\mathrm{OH}, \mathrm{R}_{3}=\mathrm{OH}$ & $\begin{array}{c}\text { S. mahagoni } \\
{[24,30,31,45,51],} \\
\text { S. macrophylla }[1] \\
\end{array}$ \\
\hline 86 & swietemahonlide & $\mathrm{R}_{1}=\mathrm{Tig}, \mathrm{R}_{2}=\mathrm{H}, \mathrm{R}_{3}=\mathrm{H}$ & S. mahagoni $[24,45]$ \\
\hline 87 & xylocarpin & $\mathrm{R}_{1}=\mathrm{AC}, \mathrm{R}_{2}=\mathrm{H}, \mathrm{R}_{3}=\mathrm{H}$ & $\begin{array}{l}\text { S. mahagoni [45], } \\
\text { S. macrophylla [49] }\end{array}$ \\
\hline 88 & humilin B & $\mathrm{R}_{1}=\mathrm{iBu}, \mathrm{R}_{2}=\mathrm{OH}, \mathrm{R}_{3}=\mathrm{H}$ & $\begin{array}{c}\text { S. humilis }[38], \\
\text { S. mahagoni }[41,45] \text {, } \\
\text { S. macrophylla }[49,58]\end{array}$ \\
\hline 89 & $\begin{array}{c}\text { humilinolide } \mathrm{A} \text { (methyl } \\
3 \beta \text {-isobutyryloxy-2,6-dihydroxy- } \\
8 \alpha, 30 \alpha \text {-epoxy-l-oxo-meliacate) }\end{array}$ & $\mathrm{R}_{1}=\mathrm{iBu}, \mathrm{R}_{2}=\mathrm{OH}, \mathrm{R}_{3}=\mathrm{OH}$ & $\begin{array}{l}\text { S. humilis }[39-41,61] \text {, } \\
\text { S. macrophylla }[58]\end{array}$ \\
\hline 90 & humilinolide B & $\mathrm{R}_{1}=\mathrm{iBu}, \mathrm{R}_{2}=\mathrm{OH}, \mathrm{R}_{3}=\mathrm{OAc}$ & S. humilis [39-41] \\
\hline 91 & humilinolide F & $\mathrm{R}_{1}=\mathrm{Tig}, \mathrm{R}_{2}=\mathrm{OAc}, \mathrm{R}_{3}=\mathrm{OAc}$ & $\begin{array}{l}\text { S. humilis [41], } \\
\text { S. macrophylla [55] }\end{array}$ \\
\hline 92 & 6-deoxyswietemahonin A & $\mathrm{R}_{1}=\mathrm{COEt}, \mathrm{R}_{2}=\mathrm{H}, \mathrm{R}_{3}=\mathrm{H}$ & S. macrophylla [48] \\
\hline 93 & swielimonoid C & $\mathrm{R}_{1}=\mathrm{Piv}, \mathrm{R}_{2}=\mathrm{H}, \mathrm{R}_{3}=\mathrm{OH}$ & S. macrophylla [60] \\
\hline 94 & $\begin{array}{c}\text { methyl } \\
3 \beta \text {-acetoxy-2,6-dihydroxy- } 8 \alpha, 30 \alpha- \\
\text { epoxy-l-oxo-meliacate }\end{array}$ & $\mathrm{R}_{1}=\mathrm{Ac}, \mathrm{R}_{2}=\mathrm{OH}, \mathrm{R}_{3}=\mathrm{OH}$ & S. macrophylla [58] \\
\hline
\end{tabular}


Table 3. Cont.

\begin{tabular}{|c|c|c|c|}
\hline No. & Compounds & Substitution Groups & Sources \\
\hline 95 & $\begin{array}{l}\text { methyl 3 } 3 \text {-tigloyloxy-2-hvdroxy- } 8 \alpha, 30 \alpha \text { - } \\
\text { epoxy-1-oxo-meliacate }\end{array}$ & $\mathrm{R}_{1}=\mathrm{Tig}, \mathrm{R}_{2}=\mathrm{OH}, \mathrm{R}_{3}=\mathrm{H}$ & $\begin{array}{l}\text { S. macrophylla }[14,58] \\
\text { S. mahagoni }[62]\end{array}$ \\
\hline 96 & 6-O-acetylswietemahonin G & $\mathrm{R}_{1}=\mathrm{Tig}, \mathrm{R}_{2}=\mathrm{OH}, \mathrm{R}_{3}=\mathrm{OAc}$ & $\begin{array}{l}\text { S. macrophylla [14], } \\
\text { S. mahagoni [62] }\end{array}$ \\
\hline 97 & $\begin{array}{l}\text { 2-acetoxyswietemahonlide } \\
\text { (swietemacrophin) }\end{array}$ & $\mathrm{R}_{1}=\mathrm{Tig}, \mathrm{R}_{2}=\mathrm{OAc}, \mathrm{R}_{3}=\mathrm{H}$ & S. macrophylla [55] \\
\hline 98 & humilinolide $\mathrm{H}$ & $\mathrm{R}_{1}=\mathrm{iBu}, \mathrm{R}_{2}=\mathrm{OAc}, \mathrm{R}_{3}=\mathrm{H}$ & S. humilis [42] \\
\hline
\end{tabular}

\subsection{Phragmalin-Type Limonoids}

Totally, 55 phragmalin-type limonoids, 99-153, have been reported from S. mahagoni and S. macrophylla. Among them, 27 novel phragmalin-type limonoids, swietenitins A-X (99-103, 106-107, 114-116, 121-125, 127-134), 2,11-diacetoxyswietenialide D (108), 11-deoxyswietenialide D (109), 2-acetoxyswietenialide D (110), together with a known compound, epoxyfebrinin B (126), were published successively in 2009 [63] and 2011 [64]. Moreover, swietephragmins A-F (135-141) were obtained from S. mahagoni [30] and swietephragmins H-J (149-151) were found in S. macrophylla [65]. In 2008, compounds 142-147 were isolated from S. macrophylla [66]. Thereafter, compounds 148 and 153 with similar structures have been reported [14,31] (Figure 5, Table 4).

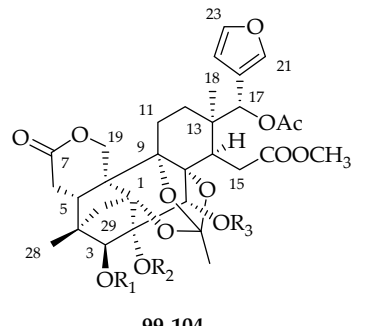

99-104

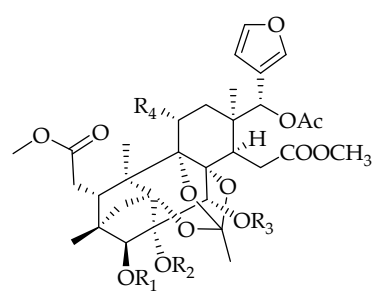

105-110

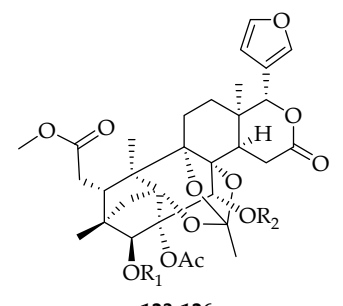

123-126

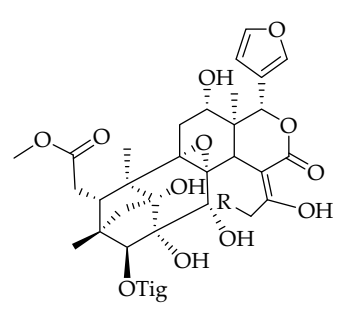

133-134

127

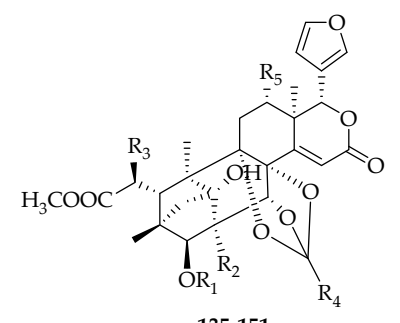

135-151
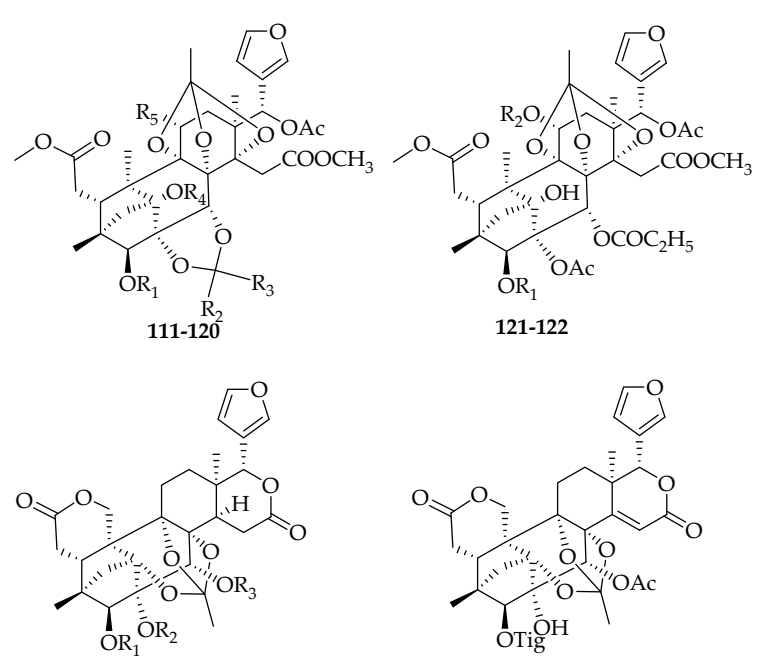

128-131
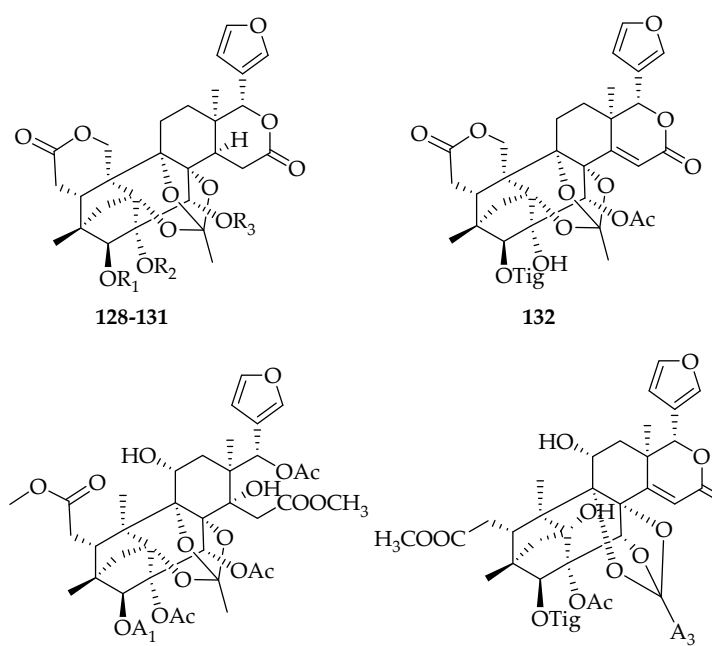

152

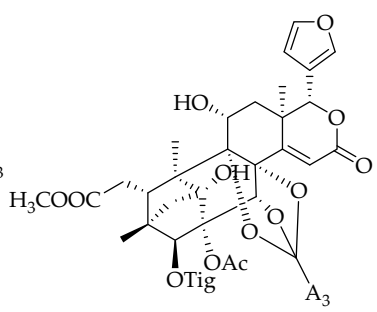

153<smiles>CC1CC2(C)OC1(C)C(=O)C2[In]</smiles><smiles>CC1=C(C)C(=O)CC(C)C1</smiles>

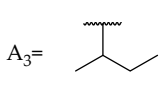

Figure 5. Chemical structures of phragmalin-type limonoids 99-153. 
Table 4. Structures and sources of phragmalin-type limonoids 99-153.

\begin{tabular}{|c|c|c|c|}
\hline No. & Compounds & Substitution Groups & Sources \\
\hline 99 & swietenitin A & $\mathrm{R}_{1}=\mathrm{A}_{1}, \mathrm{R}_{2}=\mathrm{Ac}, \mathrm{R}_{3}=\mathrm{Ac}$ & S. macrophylla [63] \\
\hline 100 & swietenitin B & $\mathrm{R}_{1}=\mathrm{A}_{2}, \mathrm{R}_{2}=\mathrm{Ac}, \mathrm{R}_{3}=\mathrm{Ac}$ & S. macrophylla [63] \\
\hline 101 & swietenitin C & $\mathrm{R}_{1}=\mathrm{A}_{1}, \mathrm{R}_{2}=\mathrm{Ac}, \mathrm{R}_{3}=\mathrm{COEt}$ & S. macrophylla [63] \\
\hline 102 & swietenitin D & $\mathrm{R}_{1}=\mathrm{A}_{1}, \mathrm{R}_{2}=\mathrm{H}, \mathrm{R}_{3}=\mathrm{COEt}$ & S. macrophylla [63] \\
\hline 103 & swietenitin E & $\mathrm{R}_{1}=\mathrm{Tig}, \mathrm{R}_{2}=\mathrm{Ac}, \mathrm{R}_{3}=\mathrm{COEt}$ & S. macrophylla [63] \\
\hline 104 & swietenitin F & $\mathrm{R}_{1}=\mathrm{Tig}, \mathrm{R}_{2}=\mathrm{H}, \mathrm{R}_{3}=\mathrm{iBu}$ & S. macrophylla [63] \\
\hline 105 & swietenialide D & $\mathrm{R}_{1}=\mathrm{A}_{1}, \mathrm{R}_{2}=\mathrm{H}, \mathrm{R}_{3}=\mathrm{COEt}, \mathrm{R}_{4}=\mathrm{OH}$ & S. mahagoni [36] \\
\hline 106 & swietenitin G & $\mathrm{R}_{1}=\mathrm{A}_{1}, \mathrm{R}_{2}=\mathrm{Ac}, \mathrm{R}_{3}=\mathrm{Ac}, \mathrm{R}_{4}=\mathrm{OH}$ & S. macrophylla [63] \\
\hline 107 & swietenitin $\mathrm{H}$ & $\mathrm{R}_{1}=\mathrm{Tig}, \mathrm{R}_{2}=\mathrm{Ac}, \mathrm{R}_{3}=\mathrm{COEt}, \mathrm{R}_{4}=\mathrm{OAc}$ & S. macrophylla [63] \\
\hline 108 & 2,11-diacetoxyswietenialide D & $\mathrm{R}_{1}=\mathrm{A}_{1}, \mathrm{R}_{2}=\mathrm{Ac}, \mathrm{R}_{3}=\mathrm{COEt}, \mathrm{R}_{4}=\mathrm{OAc}$ & S. macrophylla [63] \\
\hline 109 & 11-deoxyswietenialide D & $\mathrm{R}_{1}=\mathrm{A}_{1}, \mathrm{R}_{2}=\mathrm{H}, \mathrm{R}_{3}=\mathrm{COEt}, \mathrm{R}_{4}=\mathrm{H}$ & S. macrophylla [63] \\
\hline 110 & 2-acetoxyswietenialide D & $\mathrm{R}_{1}=\mathrm{A}_{1}, \mathrm{R}_{2}=\mathrm{Ac}, \mathrm{R}_{3}=\mathrm{COEt}, \mathrm{R}_{4}=\mathrm{OH}$ & S. macrophylla [63] \\
\hline 111 & swietenialide A & $\mathrm{R}_{1}=\mathrm{Tig}, \mathrm{R}_{2}=\mathrm{Me}, \mathrm{R}_{3}=\mathrm{OMe}, \mathrm{R}_{4}=\mathrm{H}, \mathrm{R}_{5}=\mathrm{OH}$ & S. mahagoni [36] \\
\hline 112 & swietenialide B & $\mathrm{R}_{1}=\mathrm{Tig}, \mathrm{R}_{2}=\mathrm{Et}, \mathrm{R}_{3}=\mathrm{OMe}, \mathrm{R}_{4}=\mathrm{H}, \mathrm{R}_{5}=\mathrm{OH}$ & S. mahagoni [36] \\
\hline 113 & swietenialide C & $\mathrm{R}_{1}=\mathrm{A}_{1}, \mathrm{R}_{2}=\mathrm{Me}, \mathrm{R}_{3}=\mathrm{OMe}, \mathrm{R}_{4}=\mathrm{H}, \mathrm{R}_{5}=\mathrm{OH}$ & S. mahagoni [36] \\
\hline 114 & swietenitin I & $\mathrm{R}_{1}=\mathrm{A}_{1}, \mathrm{R}_{2}=\mathrm{Et}, \mathrm{R}_{3}=\mathrm{OMe}, \mathrm{R}_{4}=\mathrm{H}, \mathrm{R}_{5}=\mathrm{OH}$ & S. macrophylla [63] \\
\hline 115 & swietenitin J & $\mathrm{R}_{1}=\mathrm{A}_{1}, \mathrm{R}_{2}=\mathrm{Et}, \mathrm{R}_{3}=\mathrm{OMe}, \mathrm{R}_{4}=\mathrm{Ac}, \mathrm{R}_{5}=\mathrm{OH}$ & S. macrophylla [63] \\
\hline 116 & swietenitin K & $\mathrm{R}_{1}=\mathrm{Tig}, \mathrm{R}_{2}=\mathrm{Et}, \mathrm{R}_{3}=\mathrm{OMe}, \mathrm{R}_{4}=\mathrm{Ac}, \mathrm{R}_{5}=\mathrm{OH}$ & S. macrophylla [63] \\
\hline 117 & swielimonoid D & $\mathrm{R}_{1}=\mathrm{A}_{1}, \mathrm{R}_{2}=\alpha-\mathrm{Et}, \mathrm{R}_{3}=\beta-\mathrm{OMe}, \mathrm{R} 4=\mathrm{Ac}, \mathrm{R}_{5}=\mathrm{OAc}$ & S. macrophylla [60] \\
\hline 118 & swielimonoid E & $\mathrm{R}_{1}=\mathrm{A}_{1}, \mathrm{R}_{2}=\beta-\mathrm{Et}, \mathrm{R}_{3}=\alpha-\mathrm{OMe}, \mathrm{R}_{4}=\mathrm{Ac}, \mathrm{R}_{5}=\mathrm{OAc}$ & S. macrophylla [60] \\
\hline 119 & swielimonoid F & $\mathrm{R}_{1}=\mathrm{A}_{1}, \mathrm{R}_{2}=\beta-\mathrm{Et}, \mathrm{R}_{3}=\alpha-\mathrm{OMe}, \mathrm{R}_{4}=\mathrm{H}, \mathrm{R}_{5}=\mathrm{OAc}$ & S. macrophylla [60] \\
\hline 120 & swielimonoid G & $\mathrm{R}_{1}=\mathrm{A}_{1}, \mathrm{R}_{2}=\beta-\mathrm{Me}, \mathrm{R}_{3}=\alpha-\mathrm{OMe}, \mathrm{R}_{4}=\mathrm{Ac}, \mathrm{R}_{5}=\mathrm{OAc}$ & S. macrophylla [60] \\
\hline 121 & swietenitin L & $\mathrm{R}_{1}=\mathrm{A}_{1}, \mathrm{R}_{2}=\mathrm{H}$ & S. macrophylla [63] \\
\hline 122 & swietenitin $\mathrm{M}$ & $\mathrm{R}_{1}=\mathrm{A}_{1}, \mathrm{R}_{2}=\mathrm{Ac}$ & S. macrophylla [63] \\
\hline 123 & swietenitin $\mathrm{N}$ & $\mathrm{R}_{1}=\mathrm{A}_{2}, \mathrm{R}_{2}=\mathrm{COEt}$ & S. macrophylla [64] \\
\hline 124 & swietenitin $\mathrm{O}$ & $\mathrm{R}_{1}=\mathrm{A}_{2}, \mathrm{R}_{2}=\mathrm{Ac}$ & S. macrophylla [64] \\
\hline 125 & swietenitin P & $\mathrm{R}_{1}=\mathrm{Tig}, \mathrm{R}_{2}=\mathrm{COEt}$ & S. macrophylla [64] \\
\hline 126 & epoxyfebrinin B & $\mathrm{R}_{1}=\mathrm{A}_{1}, \mathrm{R}_{2}=\mathrm{Ac}$ & S. macrophylla [64] \\
\hline 127 & swietenitin Q & & S. macrophylla [64] \\
\hline 128 & swietenitin $\mathrm{R}$ & $\mathrm{R}_{1}=\mathrm{A}_{1}, \mathrm{R}_{2}=\mathrm{H}, \mathrm{R}_{3}=\mathrm{COEt}$ & S. macrophylla [64] \\
\hline 129 & swietenitin S & $\mathrm{R}_{1}=\mathrm{Tig}, \mathrm{R}_{2}=\mathrm{Ac}, \mathrm{R}_{3}=\mathrm{COEt}$ & S. macrophylla [64] \\
\hline 130 & swietenitin $\mathrm{T}$ & $\mathrm{R}_{1}=\mathrm{A}_{1}, \mathrm{R}_{2}=\mathrm{H}, \mathrm{R}_{3}=\mathrm{COEt}$ & S. macrophylla [64] \\
\hline 131 & swietenitin $\mathrm{U}$ & $\mathrm{R}_{1}=\mathrm{Tig}, \mathrm{R}_{2}=\mathrm{H}, \mathrm{R}_{3}=\mathrm{Ac}$ & S. macrophylla [64] \\
\hline 132 & swietenitin $\mathrm{V}$ & & S. macrophylla [64] \\
\hline 133 & swietenitin W & $\mathrm{R}=\mathrm{H}$ & S. macrophylla [64] \\
\hline 134 & swietenitin X & $\mathrm{R}=\mathrm{Me}$ & S. macrophylla [64] \\
\hline 135 & swietephragmin A & $\mathrm{R}_{1}=\mathrm{Tig}, \mathrm{R}_{2}=\mathrm{OAc}, \mathrm{R}_{3}=\mathrm{H}, \mathrm{R}_{4}=\mathrm{iPr}, \mathrm{R}_{5}=\mathrm{H}$ & S. mahagoni [30] \\
\hline 136 & swietephragmin B & $\mathrm{R}_{1}=\mathrm{Tig}, \mathrm{R}_{2}=\mathrm{OAc}, \mathrm{R}_{3}=\mathrm{H}, \mathrm{R}_{4}=\mathrm{A}_{3}, \mathrm{R}_{5}=\mathrm{H}$ & S. mahagoni [30] \\
\hline 137 & swietephragmin C & $\mathrm{R}_{1}=\mathrm{Tig}, \mathrm{R}_{2}=\mathrm{OH}, \mathrm{R}_{3}=\mathrm{H}, \mathrm{R}_{4}=\mathrm{A}_{3}, \mathrm{R}_{5}=\mathrm{H}$ & S. mahagoni [30] \\
\hline 138 & swietephragmin D & $\mathrm{R}_{1}=\mathrm{Tig}, \mathrm{R}_{2}=\mathrm{OH}, \mathrm{R}_{3}=\mathrm{H}, \mathrm{R}_{4}=\mathrm{iPr}, \mathrm{R}_{5}=\mathrm{H}$ & S. mahagoni [30] \\
\hline 139 & swietephragmin E & $\mathrm{R}_{1}=\mathrm{Tig}, \mathrm{R}_{2}=\mathrm{OH}, \mathrm{R}_{3}=\mathrm{OH}, \mathrm{R}_{4}=\mathrm{A}_{3}, \mathrm{R}_{5}=\mathrm{H}$ & S. mahagoni [30] \\
\hline 140 & swietephragmin F & $\mathrm{R}_{1}=\mathrm{Tig}, \mathrm{R}_{2}=\mathrm{OH}, \mathrm{R}_{3}=\mathrm{H}, \mathrm{R}_{4}=\mathrm{Et}, \mathrm{R}_{5}=\mathrm{H}$ & S. mahagoni [30] \\
\hline 141 & swietephragmin G & $\mathrm{R}_{1}=\mathrm{Tig}, \mathrm{R}_{2}=\mathrm{OH}, \mathrm{R}_{3}=\mathrm{H}, \mathrm{R}_{4}=\mathrm{Me}, \mathrm{R}_{5}=\mathrm{H}$ & S. mahagoni [30] \\
\hline 142 & 6-O-acetylswietephragmin E & $\mathrm{R}_{1}=\mathrm{Tig}, \mathrm{R}_{2}=\mathrm{OH}, \mathrm{R}_{3}=\mathrm{OAc}, \mathrm{R}_{4}=\mathrm{A}_{3}, \mathrm{R}_{5}=\mathrm{H}$ & S. macrophylla [66] \\
\hline 143 & $12 \alpha$-acetoxyswietephragmin C & $\mathrm{R}_{1}=\mathrm{Tig}, \mathrm{R}_{2}=\mathrm{OH}, \mathrm{R}_{3}=\mathrm{H}, \mathrm{R}_{4}=\mathrm{A}_{3}, \mathrm{R}_{5}=\mathrm{OAc}$ & S. macrophylla [66] \\
\hline 144 & $\begin{array}{c}\text { 3ß-O-destigloyl-3 } \beta \text {-O-benzoyl-6- } \\
O \text {-acetylswietephragmin E }\end{array}$ & $\mathrm{R}_{1}=\mathrm{Bz}, \mathrm{R}_{2}=\mathrm{OH}, \mathrm{R}_{3}=\mathrm{OAc}, \mathrm{R}_{4}=\mathrm{A}_{3}, \mathrm{R}_{5}=\mathrm{H}$ & S. macrophylla [66] \\
\hline
\end{tabular}


Table 4. Cont.

\begin{tabular}{|c|c|c|c|}
\hline No. & Compounds & Substitution Groups & Sources \\
\hline 145 & 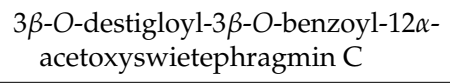 & $\mathrm{R}_{1}=\mathrm{Bz}, \mathrm{R}_{2}=\mathrm{OH}, \mathrm{R}_{3}=\mathrm{H}, \mathrm{R}_{4}=\mathrm{A}_{3}, \mathrm{R}_{5}=\mathrm{OAc}$ & S. macrophylla [66] \\
\hline 146 & $12 \alpha$-acetoxyswietephragmin D & $\mathrm{R}_{1}=\mathrm{Tig}, \mathrm{R}_{2}=\mathrm{OH}, \mathrm{R}_{3}=\mathrm{H}, \mathrm{R}_{4}=\mathrm{iPr}, \mathrm{R}_{5}=\mathrm{OAc}$ & S. macrophylla [66] \\
\hline 147 & 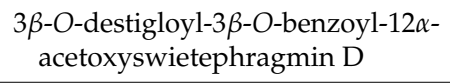 & $\mathrm{R}_{1}=\mathrm{Bz}, \mathrm{R}_{2}=\mathrm{OH}, \mathrm{R}_{3}=\mathrm{H}, \mathrm{R}_{4}=\mathrm{iPr}, \mathrm{R}_{5}=\mathrm{OAc}$ & S. macrophylla [66] \\
\hline 148 & $\begin{array}{c}\text { 6-O-acetyl-3'- } \\
\text { demethylswietephragmin E }\end{array}$ & $\mathrm{R}_{1}=\mathrm{Tig}, \mathrm{R}_{2}=\mathrm{OH}, \mathrm{R}_{3}=\mathrm{OAc}, \mathrm{R}_{4}=\mathrm{iPr}, \mathrm{R}_{5}=\mathrm{H}$ & S. macrophylla [66] \\
\hline 149 & swietephragmin $\mathrm{H}$ & $\mathrm{R}_{1}=\mathrm{Tig}, \mathrm{R}_{2}=\mathrm{OAc}, \mathrm{R}_{3}=\mathrm{H}, \mathrm{R}_{4}=\mathrm{Et}, \mathrm{R}_{5}=\mathrm{H}$ & S. macrophylla [65] \\
\hline 150 & swietephragmin I & $\mathrm{R}_{1}=\mathrm{Tig}, \mathrm{R}_{2}=\mathrm{OAc}, \mathrm{R}_{3}=\mathrm{H}, \mathrm{R}_{4}=\mathrm{Me}, \mathrm{R}_{5}=\mathrm{H}$ & S. macrophylla [65] \\
\hline 151 & swietephragmin J & $\mathrm{R}_{1}=\mathrm{Tig}, \mathrm{R}_{2}=\mathrm{OAc}, \mathrm{R}_{3}=\mathrm{H}, \mathrm{R}_{4}=\mathrm{Et}, \mathrm{R}_{5}=\mathrm{OH}$ & S. macrophylla [65] \\
\hline 152 & swietenialide E & & S. mahagoni [57] \\
\hline 153 & 11-hydroxyswietephragmin B & & S. mahogani [31] \\
\hline
\end{tabular}

\subsection{Polyoxyphragmalin-Type Limonoids}

Currently, only 11 polyoxyphragmalin-type limonoids have been isolated from Swietenia species. Among them, seven known compounds, khayanolide E (154), 1-O-acetylkhayanolide B (155), 1-O-deacetylkhayanolide E (156), khayanolide B (157), khayalactone (158), 1-O-acetylkhayanolide A (159) and khayanolide A (160), were isolated from S. macrophylla [37]. The structure of swietemahalactone (161), an example of a novel rearranged polyoxyphragmalin-type limonoid, was confirmed by X-ray crystallographic analysis [67]. Similarly, a rearrangement of the lactone ring occurred in the structure of 162 [34]. Compounds 163 and 164 were discovered from S. macrophylla in 2012 and 2009 (Figure 6, Table 5).

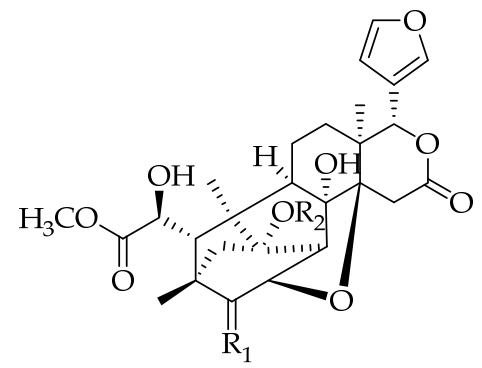

154-157

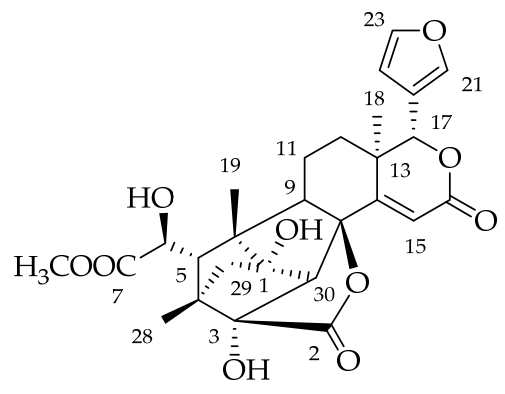

161

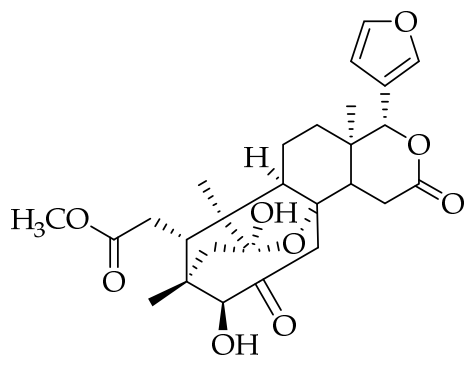

158

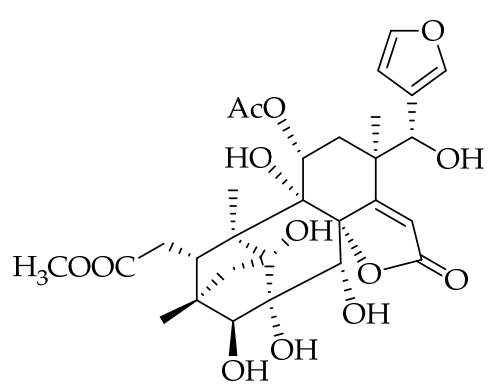

162

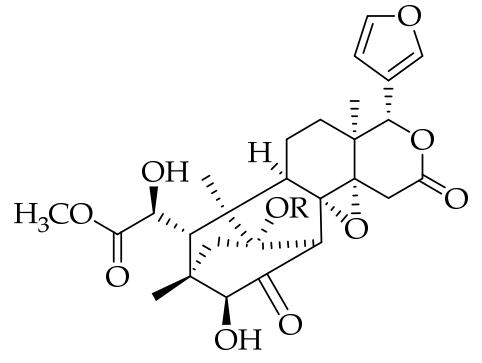

159-160

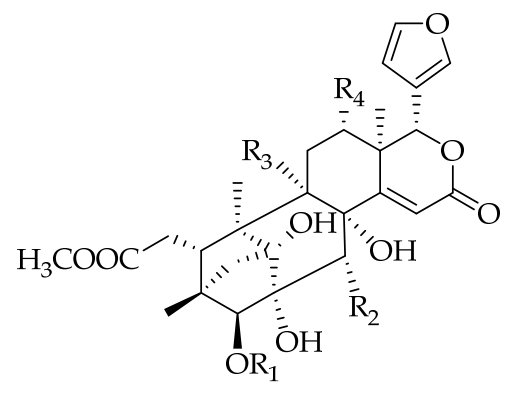

163-164

Figure 6. Chemical structures of polyoxyphragmalin-type limonoids 154-164. 
Table 5. Structures and sources of polyoxyphragmalin-type limonoids 154-164.

\begin{tabular}{|c|c|c|c|}
\hline No. & Compounds & Substitution Groups & Sources \\
\hline 154 & khayanolide E & $\mathrm{R}_{1}=\mathrm{O}, \mathrm{R}_{2}=\mathrm{Ac}$ & S. macrophylla [37] \\
\hline 155 & 1-O-acetylkhayanolide B & $\mathrm{R}_{1}=\beta-\mathrm{OH}, \alpha-\mathrm{H}, \mathrm{R}_{2}=\mathrm{Ac}$ & S. macrophylla [37] \\
\hline 156 & 1-O-deacetylkhayanolide E & $\mathrm{R}_{1}=\mathrm{O}, \mathrm{R}_{2}=\mathrm{H}$ & S. macrophylla [37] \\
\hline 157 & khayanolide B & $\mathrm{R}_{1}=\beta-\mathrm{OH}, \alpha-\mathrm{H}, \mathrm{R}_{2}=\mathrm{H}$ & S. macrophylla [37] \\
\hline 158 & khayalactone & & S. macrophylla [37] \\
\hline 159 & 1-O-acetylkhayanolide A & $\mathrm{R}=\mathrm{Ac}$ & S. macrophylla [37] \\
\hline 160 & khayanolide A & $\mathrm{R}=\mathrm{H}$ & S. macrophylla [37] \\
\hline 161 & swietemahalactone & & S. mahagoni [67] \\
\hline 162 & swiemahogin B & & S. mahagoni [34] \\
\hline 163 & swietenine J & $\mathrm{R}_{1}=\mathrm{Ac}, \mathrm{R}_{2}=\mathrm{H}, \mathrm{R}_{3}=\mathrm{H}, \mathrm{R}_{4}=\mathrm{H}$ & S. macrophylla [37] \\
\hline 164 & swietemacrophine & $\mathrm{R}_{1}=\mathrm{Tig}, \mathrm{R}_{2}=\mathrm{OTig}, \mathrm{R}_{3}=\mathrm{OH}, \mathrm{R}_{4}=\mathrm{OAc}$ & S. macrophylla [65] \\
\hline
\end{tabular}

\section{Biological Activities}

\subsection{Antifeedant Activity}

Table 6 lists the $50 \%$ antifeedant index concentration $\left(\mathrm{DC}_{50}\right)$, minimum antifeedant concentration (MAC), and antifeedant index (AI, mean \pm SEM) values of the antifeedant activity in studies using Meliaceous limonoids and Spodoptera insects. At $20 \mu \mathrm{g} /$ leaf- $\mathrm{cm}^{2}$ (1000 ppm), swietemahonin G (85) strongly inhibited the larval feeding of Spodoptera littoralis and swietephragmins 135-141 showed moderate activity [30]. Swietenialides A-E $(\mathbf{1 1 1}-\mathbf{1 1 3}, \mathbf{1 1 7}, \mathbf{1 1 8})$ showed antifeedant activity at $1000 \mathrm{ppm}$ concentration against the third-instar larvae of S. littoralis (Boisduval) [36]. Swietenolide (23), 6-O-acetylswietenolide (25), 3,6-O,O-diacetylswietenolide (27), swietenine (42), 2-hydroxyswietenine (55) and swietemahonin F (84) were evaluated at concentrations of $1000 \mathrm{ppm}$ against the final instar larvae of Spodoptera frugiperda [1]. Among these five limonoids from S. macrophylla and S. aubrevilleana, swietenine (42) showed the greatest potency with a $\mathrm{DC}_{50}$ value of $2.49 \pm 1.44(\mathrm{mg} / \mathrm{L})$. These limonoids also inhibit larval growth inhibition activity against Helicoverpa zea, Heliothis virescens and Manduca sexta insect species [68].

Table 6. Antifeedant effects of limonoids.

\begin{tabular}{|c|c|}
\hline Compounds & Insect and Antifeedant Activity \\
\hline swietenolide (23) & $\begin{array}{c}\text { Spodoptera frugiperda } \mathrm{AI}=94.1 \pm 2.90(1000 \mathrm{ppm})[1] \\
\mathrm{DC}_{50}=80.6 \pm 1.1(\mathrm{mg} / \mathrm{L})[68]\end{array}$ \\
\hline 6-acetylswietenolide (25) & S. frugiperda $\mathrm{AI}=72.2 \pm 19.60(1000 \mathrm{ppm})[1]$ \\
\hline 3,6-O,O-diacetylswietenolide (27) & S. frugiperda $\mathrm{AI}=72.0 \pm 9.38(1000 \mathrm{ppm})[1]$ \\
\hline swietemahonin F (84) & S. frugiperda $\mathrm{AI}=70.2 \pm 8.90(1000 \mathrm{ppm})[1]$ \\
\hline swietenine (42) & S. frugiperda $\mathrm{DC}_{50}=2.49 \pm 1.44(\mathrm{mg} / \mathrm{L})[68]$ \\
\hline 2-hydroxyswietenine (55) & S. frugiperda $\mathrm{DC}_{50}=65.8 \pm 1.2(\mathrm{mg} / \mathrm{L})[68]$ \\
\hline swietemahonin G (85) & S. frugiperda $\mathrm{DC}_{50}=13.8 \pm 1.2(\mathrm{mg} / \mathrm{L})[68]$, \\
\hline 3,6-O,O-diacetylswietenolide (27) & $\begin{array}{l}\text { Spodoptera littoralis, MAC values }=300[31] \\
\text { S. frugiperda, } \mathrm{DC}_{50}=4.65 \pm 1.33(\mathrm{mg} / \mathrm{L})[68]\end{array}$ \\
\hline 6-O-acetylswietemahonin G (96) & S. littoralis, MAC values $=500$ [62] \\
\hline swietenialides A-E $(\mathbf{1 1 1}-\mathbf{1 1 3}, \mathbf{1 1 7}, \mathbf{1 1 8})$ & S. littoralis, MAC values $=1000[36]$ \\
\hline 7-deacetoxy-7-oxogedunin (5) & S. littoralis, MAC values = $1000[31]$ \\
\hline methyl 6-hydroxyangolensate (16) & S. littoralis, MAC values $=500[31]$ \\
\hline 6-O-acetyl-2-hydroxyswietenin (52) & S. littoralis, MAC values $=500[31]$ \\
\hline 2-hydroxy-6-deacetoxyswietenine (54) & S. littoralis, MAC values $=500[31]$ \\
\hline 2-hydroxyswietenine (55) & S. littoralis, MAC values $=500[31]$ \\
\hline swietephragmin H (149) & S. littoralis, MAC values $=1000[31]$ \\
\hline swietephragmin I (150) & S. littoralis, MAC values $=500[31]$ \\
\hline 11-hydroxyswietephragmin B (153) & S. littoralis, MAC values = $1000[31]$ \\
\hline humilinolide B (90) & Sitophilus oryzae, $\mathrm{AI}=79.7 \pm 16.7$ [69] \\
\hline humilinolide C (50) & S. oryzae, $\mathrm{AI}=24.8 \pm 1.0[69]$ \\
\hline humilinolide D (51) & S. oryzae, $\mathrm{AI}=65.2 \pm 11.1[69]$ \\
\hline
\end{tabular}




\subsection{Antimicrobial Activity}

Eleven limonoids from Swietenia species were tested for antifungal activity against the groundnut rust Puccinia arachidis. Activity was calculated as the percent reduction in the numbers of rust pustules on treated groundnut leaflets compared with untreated control leaflets. Among these compounds, 6-acetylswietenine (48), 6-acetyl-3-tigloylswietenolide (26), 2,3-dihydroxy-3-deoxy-mexicanolide (37), $3 \beta$-hydroxymexicanolide (30), 3 $\beta$-acetoxymexicanolide (33) and mexicanolide (22) showed the highest activity, causing $80-95 \%$ reduction at $10 \mu \mathrm{g} / \mathrm{cm}^{2}$ leaflet area, while 3,6-O,O-diacetylswietenolide (27) and swietenolide (23) exhibited moderate activity, causing over $60 \%$ reduction at the same concentration. Surprisingly, swietenine (42) increased the disease severity considerably at lower concentrations relative to control [46]. The antifungal effects of ten limonoids were determined by a radial growth technique. At a concentration of $1500 \mathrm{mg} / \mathrm{L}$, deacetoxy-7-oxogedunin (5) inhibited Botrytis cinerea growth by $60.8 \%$. This value was comparable with those found with swietenine (42) at $1000 \mathrm{mg} / \mathrm{L}(57.5 \%)$ and 3-O-acetylswietenolide (24) at $1500 \mathrm{mg} / \mathrm{L}$ (63.1\%) [70]. 2-Hydroxy-3-O-tigloylswietenolide (31) and swietenolide (23) were tested against eight multiple-drug-resistant bacterial strains using the conventional agar disc diffusion assay. The former compound exhibited more potent antimicrobial activity than the latter compound against all tested fungi (Group A $\beta$ haemolytic Streptococcus aureus, Staphylococcus aureus, Streptococcus pneumoniae, Haemophilus influenzae, Escherichia coli, Klebsiella pneumoniae, Salmonella typhi, and Salmonella paratyphi) Vancomycin $(10 \mu \mathrm{g} / \mathrm{disc})$ was used as the positive control. [47]. Thirty limonoids from S. mahagoni were inactive in antimicrobial testing against 11 microbes (seven bacteria and four fungi) in vitro. However, 2-hydroxy-3-O-isobutyrylproceranolide (34) and 2-hydroxyfissinolide (36) exhibited activity against Micrococcus luteus ATCC 9341 with MIC values of 50 and $12.5 \mu \mathrm{g} / \mathrm{mL}$, respectively, in a broth dilution test. Ofloxacin was used as the positive control [29].

\subsection{Hypoglycemic Activity}

When assayed for effects on peripheral glucose utilization employing an isolated rat hemidiaphragm method, swietenine (42) exhibited significant $(p<0.01)$ activity comparable with that of human insulin $(p<0.01)$ [71]. In the same year, the same compound was also found to exhibit significant dose-dependent hypoglycemic and hypolipidemic activity in type 2 diabetic rats when given by oral administration at 25 and $50 \mathrm{mg} / \mathrm{kg}$ body weight per day [72]. Dewanjee et al. obtained similar conclusions in 2011 [73]. Compounds 60, 54 and 88 were active $(3.16-31.6 \mathrm{mg} / \mathrm{kg}$, bw) when tested as hypoglycemic agents in normal and NA-STZ-hyperglycemic mice [42]. Three S. macrophylla bioactive compounds, 6-O-acetylswietenolide (25), 3,6-O,O-diacetylswietenolide (27), and swietenine (42), induced uptake of glucose by muscle cells by increasing the translocation of GLUT4 to the plasma membrane. The limonoids exhibited a good potential for anti-diabetic activity, however, with a minimal side effect of weight gain [33].

\subsection{Anti-PAF Activity}

Kadota et al. published the first example of limonoids having antagonistic effects on PAF, finding the following rank order of inhibition at $100 \mu \mathrm{g} / \mathrm{mL}$ : swietemahonin A (79), 97.4\%; swietemahonin E (83), 91.7\%; 3-O-acetylswietenolide (24), 91.6\%; swietenolide (23), 35.2\% [52]. In other examples, swietemahonins A, D, E, G (79, 81-82, 85), 3-O-acetylswietenolide (24) and 6-O-acetylswietenolide (25), strongly inhibited PAF-induced aggregation of rabbit platelets in vitro, giving $\mathrm{IC}_{50}$ values of $40.2,40.3,51.2,42.6,52.9,80.4$ and $55.6 \mu \mathrm{g} / \mathrm{mL}$. The same study reported that swietemahonin E (83) reduced PAF-induced mortality in mice [51].

\subsection{Anti-Inflammatory Activities}

6-O-Acetyl-3'-demethylswietephragmin E (148), 3,6-O,O-diacetylswietenolide (27), 3-O-tigloylswietenolide (28), 3-O-tigloyl-6-O-acetylswietenolide (26), swietemahonin E (83), methyl 3 $\beta$-tigloyloxy- 
2-hydroxy- $8 \alpha, 30 \alpha$-epoxy-l-oxomeliacate (95), and 6-O-acetylswietemahonin G (96) inhibited formyl-Lmethionyl-L-leucyl-L-phenylalanine (fMLP)-induced superoxide anion generation with $\mathrm{IC}_{50}$ values of $27.6-48.7 \mu \mathrm{M}$. The assay was based on the superoxide dismutaste (SOD)-inhibitable reduction of ferricytochrome $c$ and used ibuprofen as the positive control. Among all tested compounds, $\mathbf{9 6}$ was the most potent against $\mathrm{O}_{2}^{--}$generation. A $8 \alpha, 30 \alpha$-epoxy group was beneficial, and acetyl substitution at C-6 was preferable to hydroxy or no substitution [14]. In addition, swietemacrophin (97) and humilinolide F (91) exhibited moderate activity with $\mathrm{IC}_{50}$ values of 45.44 and $27.13 \mu \mathrm{g} / \mathrm{mL}$ [56].

\subsection{Other Activities}

Limonoids 7, 15, 23, 48, and 92 were tested for their in vitro half-maximal effective concentration against dengue virus 2 and showed inhibitory activity in the concentration range of 3.5 to $12.5 \mu \mathrm{M}$. Among the five limonoids, 92 exhibited significant antiviral activity $\left(\mathrm{EC}_{50}=7.2 \pm 1.33 \mu \mathrm{M}\right)$ with a selectivity index $\left(\mathrm{CC}_{50} / \mathrm{EC}_{50}\right)$ value greater than 27.7 [35]. Swieteliacate B (3) was moderately active against HL-60 and SW-480 with IC $_{50}$ values of 30.59 and $32.68 \mu \mathrm{M}$ [26]. 7-Deacetoxy-7-oxogedunin (5) was cytotoxic toward Hep-G2 cells with an $\mathrm{IC}_{50}$ value of $16.17 \mu \mathrm{M}$ [74]. Humilinolides A-D (89-90, 50-51) showed weak cytotoxic activity against three human tumor cell lines (A-549, MCF-7 and HT-29), and generally produced high mortality rates against larvae of Ostrinia nubilalis [40]. Similarly, when tested against the growth of O. nubilalis, humilinolide E (52) and methyl-2-hydroxy-3 $\beta$-isobutyroxy-1-oxomeliac-8(30)-enate (53) showed comparable effects to those of the positive control, toosendandin, in terms of reduction of \% pupation and \% adult emergence, while humilin B (88) and swietenine $C$ were effective only for adult emergence [41]. Five limonoids, swietenolide (23), 3,6-O,O-diacetylswietenolide (27), swietenine (42), swietemahonin G (85), and 2-hydroxyswietenine (55), isolated from S. macrophylla and S. aubrevilleana, were tested in the Artemia salina lethality assay. Only 85 showed weak activity ( $\mathrm{LC}_{50} 220.1 \mathrm{ppm}$ ); however, certain semi-synthetic structural modifications led to increased toxicity. The addition of acyl groups, particularly benzoyl groups, was quite effective; for example, 6-O-benzoylswietenolide ( $\mathrm{LC}_{50} 4.3 \mathrm{ppm}$ ) and 6-O-benzoylswietenine $\left(\mathrm{LC}_{50} 7.5 \mathrm{ppm}\right)$ were significantly more active than the non-acylated parent compounds 23 and 42, respectively $\left(\mathrm{LC}_{50}>500 \mathrm{ppm}\right.$ ) [75]. Humilinolide A (89) can cause intestinal spasmogenic and uterotonic action [61]. Swietephragmin $\mathrm{H}(\mathbf{1 4 9})$ and swietephragmin I (150) possessed low anti-oxidative effects (17.12 $\pm 0.49 \%$ and $13.43 \pm 0.28 \%$, respectively) at the highest concentration $(320 \mu \mathrm{g} / \mathrm{mL})$ tested. These two compounds lack $\mathrm{H}$-atom donating ability and electron delocalised potential, which are important structural features for significant antioxidant potency [65]. Local injection of mexicanolide (22) $(0.5-3.5 \mathrm{mg})$ led to concentration-dependent antihyperalgesic action in NA-STZ hyperglycemic mice [76].

\section{Conclusions}

Swietenia is a genus in the subfamily mahogany (Meliaceae), which is generally considered to contain 7 to 8 species. Among them, the seeds and bark of S. mahagoni, S. macrophylla and S. humilis are used in folk medicines for the treatment of hypertension, diabetes, malaria, and epilepsy in Indonesia, India and Mexico [2,76,77]. Based on the data available, this paper summarizes five types of limonoids and describes various bioactive activities, such as antifeedant, hypoglycemic, antimicrobial, anti-PAF, anti-inflammatory, antitumor, insecticidal, anti-oxidative and antihyperalgesic. Although most of the limonoids isolated from Swietenia species do not show significant antiproliferative effects against cancer cell lines, some structurally similar limonoids isolated from Melia azedarach exhibit good antitumor activity. The best known compound is toosendanin (165), which strong inhibits multiple tumor cell lines; its $\mathrm{IC}_{50}$ values were $0.005,0.009$ and $0.0054 \mu \mathrm{M}$ against HL60, AZ521 and U937, respectively [78,79]. In addition, meliarachin C (166), 12-dehydro-29-exo-neoazedarachin D (167), and 1-O-cinnamoyltrichilinin (168) exhibited $\mathrm{IC}_{50}$ values ranging from 0.65 to $9.1 \mu \mathrm{M}$ against HL60 [79]. Erythrocarpine A (169), isolated from Chisocheton erythrocarpus, showed cytotoxicity against P388 murine leukemia cells with $\mathrm{IC}_{50}$ value of $2.0 \mu \mathrm{g} / \mathrm{mL}$ [80] (Figure 7). Its structure differs from that 
of seenganolide A (67) only by the presence of a benzoyl ester rather than hydroxy group. Therefore, limonoids from the genus Swietenia still have great potential for biological activity and may be modified structurally to improve their activity.

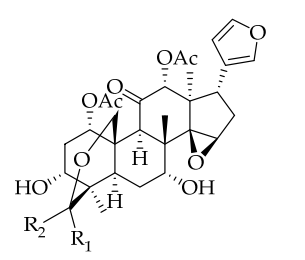

$165 \mathrm{R}_{1}=\mathrm{H}, \mathrm{R}_{2}=\mathrm{OH}$ and $\mathrm{R}_{1}=\mathrm{OH}, \mathrm{R}_{2}=\mathrm{H}$ $166 \mathrm{R}_{1}=\mathrm{H}, \mathrm{R}_{2}=\mathrm{OMe}$

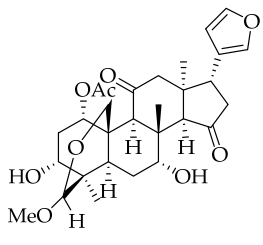

167

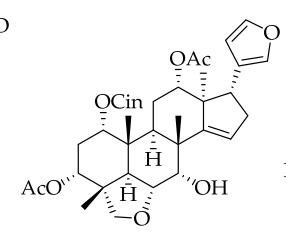

168

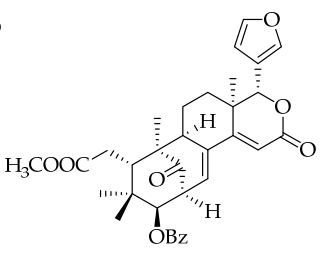

169<smiles>CC(CC(C)(C)C(F)(F)F)C(=O)C=Cc1ccccc1</smiles>

$\mathrm{Bz}=$

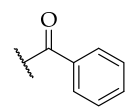

Figure 7. Chemical structures of limonoids 165-169 from other plants.

Furthermore, the published research on Swietenia has been focused mostly on the seeds and their limonoid components; however, but other plant parts and other compound types may also have rich pharmacological activities. Therefore, it is extremely urgent to expand the scope of research on Swietenia and discover or develop additional biologically active constituents of this plant genus.

Funding: Partial support was provided by NIH Grant CA177584 from the National Cancer Institute awarded to K.H.L, National Natural Science Foundation of China (81303220) and Key Research and Development Project of Anhui Province (1704a0802145).

Acknowledgments: We thank the NPRL members who assisted in and supported the review.

Conflicts of Interest: The authors declare no conflicts of interest.

\section{References}

1. Mootoo, B.S.; Ali, A.; Motilal, R.; Pingal, R.; Ramlal, A.; Khan, A.; Reynolds, W.F.; McLean, S. Limonoids from Swietenia macrophylla and S. aubrevilleana. J. Nat. Prod. 1999, 62, 1514-1517. [CrossRef] [PubMed]

2. Panda, S.P.; Haldar, P.K.; Bera, S.; Adhikary, S.; Kandar, C.C. Antidiabetic and antioxidant activity of Swietenia mahagoni in streptozotocin-induced diabetic rats. Pharm. Biol. 2010, 48, 974-979. [CrossRef] [PubMed]

3. Dutta, M.; Biswas, U.K.; Chakraborty, R.; Banerjee, P.; Raychaudhuri, U. Regeneration of pancreatic $\beta$-cells on streptozotocin induced diabetic rats under the effect of swietenia macrophylla seeds. Int. J. Green Pharm. 2013, 6, 336-339. [CrossRef]

4. Dutta, M.; Biswas, U.K.; Chakraborty, R.; Banerjee, P.; Maji, D.; Mondal, M.C.; Raychaudhuri, U. Antidiabetic and antioxidant effect of Swietenia macrophylla seeds in experimental type 2 diabetic rats. Int. J. Diabetes Dev. Ctries. 2013, 33, 60-65. [CrossRef]

5. Dewanjee, S.; Kundu, M.; Maiti, A.; Majumdar, R.; Majumdar, A.; Mandel, S.C. In vitro evaluation of antimicrobial activity of crude extract from plants Diospyros peregrina, Coccinia grandis and Swietenia macrophylla. Trop. J. Pharm. Res. 2007, 6, 773-778. [CrossRef]

6. Sahgal, G.; Ramanathan, S.; Sasidharan, S.; Mordi, M.N.; Ismail, S.; Mansor, S.M. Phytochemical and antimicrobial activity of Swietenia mahagoni crude methanolic seed extract. Trop. Biomed. 2009, 26, 274-279. [PubMed]

7. Mohammed, S.B.; Azhari, N.H.; Mashitah, Y.M.; Abdurahman, N.H.; Mazza, A.S. Physicochemical characterization and antimicrobial activity of Swietenia macrophylla King seed oil. Esrsa Publ. 2014, 3, 1787-1792.

8. Sahgal, G.; Ramanathan, S.; Sasidharan, S.; Mordi, M.N.; Ismail, S.; Mansor, S.M. In Vitro antioxidant and xanthine oxidase inhibitory activities of methanolic Swietenia mahagoni seed extracts. Molecules 2009, 14, 4476-4485. [CrossRef]

9. Pamplona, S.; Sã, P.; Lopes, D.; Costa, E.; Yamada, E.; Silva, C.; Arruda, M.; Souza, J.; da Silva, M. In vitro cytoprotective effects and antioxidant capacity of phenolic compounds from the leaves of Swietenia macrophylla. Molecules 2015, 20, 18777-18788. [CrossRef] [PubMed] 
10. Maiti, A.; Dewanjee, S.; Mandal, S.C. In vivo evaluation of antidiarrhoeal activity of the seed of Swietenia macrophylla King (Meliaceae). Trop. J. Pharm. Res. 2007, 6, 711-716. [CrossRef]

11. Goh, B.H.; Kadir, H.A. In vitro cytotoxic potential of Swietenia macrophylla King seeds against human carcinoma cell lines. J. Med. Plant. Res. 2011, 5, 1395-1404.

12. Goh, B.H.; Chan, C.K.; Kamarudin, M.N.A.; Kadir, H.A. Swietenia macrophylla King induces mitochondrial-mediated apoptosis through p53 upregulation in HCT116 colorectal carcinoma cells. J. Ethnopharmacol. 2014, 153, 375-385. [CrossRef] [PubMed]

13. Zalabani, S.M.E.; El-Askary, H.I.; Mousa, O.M.; Issa, M.Y.; Zaitoun, A.A.; Abdel-Sattar, E. Acaricidal activity of Swietenia mahogani and Swietenia macrophylla, ethanolic extracts against Varroa destructor in honeybee colonies. Exp. Parasitol. 2012, 130, 166-170. [CrossRef] [PubMed]

14. Chen, J.J.; Huang, S.S.; Liao, C.H.; Wei, W.C.; Sung, P.J.; Wang, T.C.; Cheng, M.J. A new phragmalin-type limonoid and anti-inflammatory constituents from the fruits of Swietenia macrophylla. Food Chem. 2010, 120, 379-384. [CrossRef]

15. Wu, S.F.; Lin, C.K.; Chuang, Y.S.; Chang, F.R.; Tseng, C.K.; Wu, Y.C.; Lee, J.C. Anti-hepatitis C virus activity of 3-hydroxy caruilignan C from Swietenia macrophylla stems. J. Viral Hepat. 2012, 19, 364-370. [CrossRef] [PubMed]

16. Falah, S.; Suzuki, T.; Katayama, T. Chemical constituents from Swietenia macrophylla bark and their antioxidant activity. Pak. J. Biol. Sci. 2008, 11, 2007-2012. [CrossRef] [PubMed]

17. Soares, M.G.; Batista-Pereira, L.G.; Fernandes, J.B.; Corrêa, A.G.; Da, S.M.; Vieira, P.C.; Filho, E.R.; Ohashi, O.S. Electrophysiological responses of female and male Hypsipyla grandella (Zeller) to Swietenia macrophylla essential oils. J. Chem. Ecol. 2003, 29, 2143-2151. [CrossRef] [PubMed]

18. Tan, Q.G.; Luo, X.D. Meliaceous limonoids: Chemistry and biological activities. Chem. Rev. 2011, 111, 7437-7522. [CrossRef] [PubMed]

19. Pereira, T.B.; Silva, L.F.R.E.; Amorim, R.C.; Melo, M.R.; Souza, R.C.Z.D.; Eberlin, M.N.; Lima, E.S.; Vasconcellos, M.C.; Pohlit, A.M. In vitro, and in vivo, anti-malarial activity of limonoids isolated from the residual seed biomass from Carapa guianensis (andiroba) oil production. Malar. J. 2014, 13, 317-324. [CrossRef] [PubMed]

20. Yan, Y.X.; Liu, J.Q.; Wang, H.W.; Chen, J.X.; Chen, J.C.; Chen, L.; Zhou, L.; Qiu, M.H. Identification and antifeedant activities of limonoids from Azadirachta indica. Chem. Biodivers. 2015, 12, 1040-1046. [CrossRef] [PubMed]

21. Ambrozin, A.R.P.; Leite, A.C.; Bueno, F.C.; Vieira, P.C.; Fernandes, J.B.; Bueno, O.C.; Silva, M.F.G.F.; Pagnocca, F.C.; Hebling, M.J.A.; Maurício, B., Jr. Limonoids from andiroba oil and Cedrela fissilis and their insecticidal activity. J. Braz. Chem. Soc. 2006, 17, 542-547. [CrossRef]

22. Hu, J.; Song, Y.; Mao, X.; Wang, Z.J.; Zhao, Q.J. Limonoids isolated from Toona sinensis, and their radical scavenging, anti-inflammatory and cytotoxic activities. J. Funct. Foods 2016, 20, 1-9. [CrossRef]

23. Kadota, S.; Marpaung, L.; Kikuchi, T.; Ekitomo, H. Mahonin and secomahoganin, new tetranortriterpenoids from Swietenia mahogani (L.) JACQ. Chem. Pharm. Bull. 1989, 37, 1419-1421. [CrossRef]

24. Kadota, S.; Marpaung, L.; Kikuchi, T.; Ekitomo, H. Constituents of the seeds of Swietenia mahagoni Jacq. I. Isolation, structures, and $1 \mathrm{H}-$ and 13C-nuclear magnetic resonance signal assignments of new tetranortriterpenoids related to swietenine and swietenolide. Chem. Pharm. Bull. 1990, 38, 639-651. [CrossRef]

25. Kadota, S.; Marpaung, L.; Kikuchi, T.; Ekitomo, H. Constituents of the seeds of Swietenia mahagoni JACQ. III. Structures of mahonin and secomahoganin. Chem. Pharm. Bull. 1990, 38, 1495-1500. [CrossRef]

26. Sun, Y.P.; Zhu, L.L.; Liu, J.S.; Yu, Y.; Zhou, Z.Y.; Wang, G.; Wang, G.K. Limonoids and triterpenoid from fruit of Swietenia macrophylla. Fitoterapia 2018, 125, 141-146. [CrossRef] [PubMed]

27. Zhang, R.; Cao, M.M.; He, H.P.; Zhang, Y.; Di, Y.T.; Hao, X.J. Limonoids from the twigs and leaves of Swietenia macrophylla. Nat. Prod. Res. Dev. 2013, 25, 969-971. [CrossRef]

28. Mi, C.N.; Mei, W.L.; Li, W.; Wang, J.; Cai, C.H.; li, S.P.; Dai, H.F. Chemical Constituents from the Roots of Swietenia macrophylla King. J. Trop. Subtrop. Bot. 2017, 25, 610-616. [CrossRef]

29. Lin, B.D.; Tao, Y.; Zhang, C.R.; Dong, L.; Zhang, B.; Wu, Y.; Yue, J.M. Structurally diverse limonoids from the fruits of Swietenia mahagoni. J. Nat. Prod. 2009, 72, 2084-2090. [CrossRef] [PubMed]

30. Abdelgaleil, S.A.M.; Doe, M.; Morimoto, Y.; Nakatani, M. Rings B,D-seco limonoids from the leaves of Swietenia mahogani. Phytochemistry 2006, 67, 452-458. [CrossRef] [PubMed] 
31. Abdelgaleil, S.A.M.; Doe, M.; Nakatani, M. Rings B, D-seco limonoid antifeedants from Swietenia mahogani. Phytochemistry 2013, 96, 312-317. [CrossRef] [PubMed]

32. Liu, J.S.; Zhu, L.L.; Wang, G.; Wang, G.K. Studies on chemical constituents from the fruit of Swietenia macrophylla. J. Chin. Med. Mater. 2016, 7, 1530-1534. [CrossRef]

33. Lau, W.K.; Goh, B.H.; Kadir, H.A.; Shu-Chien, A.C.; Muhammad, T.S.T. Potent PPAR ligands from Swietenia macrophylla are capable of stimulating glucose uptake in muscle cells. Molecules 2015, 20, 22301-22314. [CrossRef] [PubMed]

34. Chen, Y.Y.; Wang, X.N.; Fan, C.Q.; Yin, S.; Yue, J.M. Swiemahogins A and B, two novel limonoids from Swietenia mahogani. Tetrahedron Lett. 2007, 48, 7480-7484. [CrossRef]

35. Cheng, Y.B.; Chien, Y.T.; Lee, J.C.; Tseng, C.K.; Wang, H.C.; Lo, I.W.; Wu, Y.H.; Wang, S.Y.; Wu, Y.C.; Chang, F.R. Limonoids from the seeds of Swietenia macrophylla with inhibitory activity against dengue Virus 2. J. Nat. Prod. 2014, 77, 2367-2374. [CrossRef] [PubMed]

36. Saad, M.M.G.; Iwagawa, T.; Doe, M.; Nakatani, M. Swietenialides, novel ring D opened phragmalin limonoid orthoesters from Swietenia mahogani JACQ. Tetrahedron 2003, 59, 8027-8033. [CrossRef]

37. Liu, J.Q.; Wang, C.F.; Chen, J.C.; Qiu, M.H. Limonoids from the leaves of Swietenia macrophylla. Nat. Prod. Res. 2012, 26, 1887-1891. [CrossRef] [PubMed]

38. Okorie, D.A.; Taylor, D.A.H. Meliaceae: Limonoids from Swietenia humilis. Phytochemistry 1971, 10, 469-470. [CrossRef]

39. Segura-Correa, R.; Mata, R.; Anaya, A.L.; Hernandez-Bautista, B.; Viliena, R.; Soriano-Garcia, M.; Bye, R.; Linares, E. New tetranortriterpenoids from Swietenia humilis. J. Nat. Prod. 1993, 56, 1567-1574. [CrossRef]

40. Jimenez, A.; Mata, R.; Pereda-Miranda, R.; Calderon, J.; Isman, M.B.; Nicol, R.; Arnason, J.T. Insecticidal limonoids from Swietenia humilis, and Cedrela salvadorensis. J. Chem. Ecol. 1997, 23, 1225-1234. [CrossRef]

41. Jimenez, A.; Villarreal, C.; Toscano, R.A.; Cook, M.; Arnason, J.T.; Bye, R.; Mata, R. Limonoids from Swietenia humilis and Guarea grandiflora (Meliaceae). Phytochemistry 1998, 49, 1981-1988. [CrossRef]

42. Ovalle-Magallanes, B.; Medina-Campos, O.N.; Pedraza-Chaverri, J.; Mata, R. Hypoglycemic and antihyperglycemic effects of phytopreparations and limonoids from Swietenia humilis. Phytochemistry 2015, 110, 111-119. [CrossRef] [PubMed]

43. Kadota, S.; Yanagawa, K.; Kikuchi, T.; Tanaka, K. Mahagonin, a novel dimeric tetranortriterpenoid from, Swietenia mahogan JACQ. Tetrahedron Lett. 1990, 31, 5943-5946. [CrossRef]

44. Fowles, R.G.; Mootoo, B.S.; Ramsewak, R.; Reynolds, W.; Lough, A.J. 3,6-Di-O-acetyl-swietenolide 0.25-hydrate. Acta Crystallogr. 2007, 63, o660-0661. [CrossRef]

45. Kadota, S.; Marpaung, L.; Kikuchi, T.; Ekimoto, H. Constituents of the seeds of Swietenia mahagoni JACQ. II. structures of swietemahonin A, B, C, D, E, F, and G and swietemahonolide. Chem. Pharm. Bull. 1990, 38, 894-901. [CrossRef]

46. Govindachari, T.R.; Suresh, G.; Banumathy, B.; Masilamani, S.; Gopalakrishnan, G.; Kumari, G.N.K. Antifungal activity of some B,D-seco limonoids from two Meliaceous plants. J. Chem. Ecol. 1999, 25, 923-933. [CrossRef]

47. Rahman, A.K.M.S.; Chowdhury, A.K.A.; Ali, H.A.; Raihan, S.Z.; Ali, M.S.; Nahar, L.; Sarker, S.D. Antibacterial activity of two limonoids from swietenia mahagoni against multiple-drug-resistant (MDR) bacterial strains. J. Nat. Med. 2009, 63, 41-45. [CrossRef] [PubMed]

48. Ma, Y.Q.; Jiang, K.; Deng, Y.; Guo, L.; Wan, Y.Q.; Tan, C.H. Mexicanolide-type limonoids from the seeds of Swietenia macrophylla. J. Asian Nat. Prod. Res. 2017, 1-7. [CrossRef] [PubMed]

49. Taylor, A.R.H.; Taylor, D.A.H. Limonoid extractives from Swietenia macrophylla. Phytochemistry 1983, 22, 2870-2871. [CrossRef]

50. Chan, K.C.; Tang, T.S.; Toh, H.T. Isolation of swietenolide diacetate from swietenia macrophylla. Phytochemistry 1976, 15, 429-430. [CrossRef]

51. Ekimoto, H.; Irie, Y.; Araki, Y.; Han, G.Q.; Kadota, S.; Kikuchi, T. Platelet aggregation inhibitors from the seeds of Swietenia mahagoni: Inhibition of in vitro and in vivo platelet-activating factor-induced effects of tetranortriterpenoids related to swietenine and swietenolide. Planta Med. 1991, 57, 56-58. [CrossRef] [PubMed]

52. Kadota, S.; Marpaung, L.; Kikuchi, T.; Ekimoto, H. Antagonists of platelet activating factor from Swietenia mahogani (L.) JACQ. Tetrahedron Lett. 1989, 30, 1111-1114. [CrossRef] 
53. Goh, B.H.; Kadir, H.A.; Malek, S.N.A.; Ng, S.W. $(\alpha R, 4 R, 4 a R, 6 a S, 7 R, 8 S, 10 R, 11 S)$-methyl $\alpha$-acetoxy-4-(3furanyl)-10-hydroxy-4a,7,9,9-tetramethyl-2,13-dioxo-1, 4,4a,5,6,6a,7,8,9,10,11,12-dodecahydro-7,11-methano$2 \mathrm{H}$-cycloocta[f][2]benzopyran-8-acetate (6-O-acetylswietenolide) from the seeds of Swietenia macrophylla. Acta Cryst. 2010, 66, 2802-2803.

54. Goh, B.H.; Kadir, H.A.; Malek, S.N.A.; Ng, S.W. Swietenolide diacetate from the seeds of swietenia macrophylla. Acta Cryst. 2010, 66, o1396. [CrossRef]

55. Chen, L.C.; Liao, H.R.; Chen, P.Y.; Kuo, W.L.; Chang, T.H.; Sung, P.J.; Wen, Z.H.; Chen, J.J. Limonoids from the seeds of Swietenia macrophylla and their anti-inflammatory activities. Molecules 2015, 20, 18551-18564. [CrossRef] [PubMed]

56. Daily, A.; Seligmann, O.; Lotter, H.; Wagner, H. 2-Hydroxy-swietenin, ein neues limonoid aus Swietenia mahagoni DC./2-Hydroxy-swietenin, a new limonoid from Swietenia mahagoni DC. Z. Naturforsch. C 1985, 40, 519-522. [CrossRef]

57. Solomon, K.A.; Malathi, R.; Rajan, S.S.; Narasimhan, S.; Nethaji, M. Swietenine. Acta Cryst. 2003, 59, o1519-o1521. [CrossRef]

58. Kojima, K.; Isaka, K.; Ogihara, Y. Tetranortriterpenoids from Swietenia macrophylla. Chem. Pharm. Bull. 1998, 46, 523-525. [CrossRef]

59. Govindachari, T.R.; Banumathy, B.; Gopalakrishnan, G.; Suresh, G. 6-Desoxyswietenine, a tetranortriterpenoid from Swietenia mahogani. Fitoterapia 1999, 70, 106-108. [CrossRef]

60. Cheng, Y.B.; Chien, Y.T.; Lee, J.C.; Wu, Y.C.; Chang, F.R. Anti-dengue virus limonoids from the actual seeds of Swietenia macrophylla. Planta Med. 2014, 80, 311-323. [CrossRef]

61. Perusquía, M.; Herńndez, R.; Jiménez, M.A.; Pereda-Miranda, R.; Mata, R. Contractile response induced by a limonoid (humilinolide A) on spontaneous activity of isolated smooth muscle. Phytother. Res. 1997, 11, 354-357. [CrossRef]

62. Saad, M.M.G. Three new mexicanolides from the stem bark of Swietenia mahogani Jacq. Heterocycles 2004, 63, 389-399. [CrossRef]

63. Lin, B.D.; Zhang, C.R.; Yang, S.P.; Wu, Y.; Yue, J.M. D-ring-opened phragmalin-type limonoid orthoesters from the twigs of swietenia macrophylla. J. Natl. Prod. 2009, 72, 1305-1313. [CrossRef] [PubMed]

64. Lin, B.D.; Zhang, C.R.; Yang, S.P.; Wu, Y.; Yue, J.M. Phragmalin-type limonoid orthoesters from the twigs of Swietenia macrophylla. Chem. Pharm. Bull. 2011, 59, 458-465. [CrossRef] [PubMed]

65. Tan, S.K.; Osman, H.; Kengchong, W.; Penglim, B. New phragmalin-type limonoids from Swietenia macrophylla king. Food Chem. 2009, 115, 1279-1285. [CrossRef]

66. Silva, M.N.D.; Arruda, M.S.P.; Castro, K.C.F.; da Silva, M.F.D.G.; Fernandes, J.B.; Vieira, P.C. Limonoids of the phragmalin type from Swietenia macrophylla and their chemotaxonomic significance. J. Nat. Prod. 2008, 71, 1983-1987. [CrossRef] [PubMed]

67. Liu, J.Q.; Peng, X.R.; Zhang, W.M.; Shi, L.; Li, X.Y.; Chen, J.C.; Qiu, M.H. Swietemahalactone, a rearranged phragmalin-type limonoid with anti-bacterial effect, from Swietenia mahagoni. RSC Adv. 2013, 3, 4890-4893. [CrossRef]

68. Fowles, R.; Mootoo, B.; Ramsewak, R.; Khan, A.; Ramsubhag, A.; Reynolds, W.; Nair, M. Identification of new limonoids from Swietenia and their biological activity against insects. Pest Manag. Sci. 2010, 66, 1298-1303. [CrossRef] [PubMed]

69. Omar, S.; Marcotte, M.; Fields, P.; Sanchez, P.E.; Poveda, L.; Mata, R.; Jimenez, A.; Durst, T.; Zhang, J.; MacKinnon, S.; et al. Antifeedant activities of terpenoids isolated from tropical Rutales. J. Stored Prod. Res. 2007, 43, 92-96. [CrossRef]

70. Abdelgaleil, S.A.; Hashinaga, F.; Nakatani, M. Antifungal activity of limonoids from Khaya ivorensis. Pest Manag. Sci. 2005, 61, 186-190. [CrossRef] [PubMed]

71. Maiti, A.; Dewanjee, S.; Sahu, R. Isolation of hypoglycemic phytoconstituent from Swietenia macrophylla seeds. Phytother. Res. 2009, 23, 1731-1733. [CrossRef] [PubMed]

72. Dewanjee, S.; Maiti, A.; Das, A.K.; Mandal, S.C.; Dey, S.P. Swietenine: A potential oral hypoglycemic from Swietenia macrophylla seed. Fitoterapia 2009, 80, 249-251. [CrossRef] [PubMed]

73. Maiti, A. Swietenine, big leaf mahogany (Swietenia macrophylla) seed extract as a hypoglycemic agent. In Nuts and Seeds in Health and Disease Prevention; Elsevier Inc.: New York, NY, USA, 2011; pp. 205-212. 
74. Pudhom, K.; Sommit, D.; Nuclear, P.; Ngamrojanavanich, N.; Petsom, A. Protoxylocarpins F-H, protolimonoids from seed kernels of Xylocarpus granatum. J. Nat. Prod. 2009, 72, 2188-2191. [CrossRef] [PubMed]

75. Fowles, R.G.; Mootoo, B.S.; Ramsewak, R.S.; Khan, A. Toxicity-structure activity evaluation of limonoids from Swietenia species on Artemia salina. Pharm. Biol. 2012, 50, 264-267. [CrossRef] [PubMed]

76. Ovallemagallanes, B.; Décigacampos, M.; Mata, R. Antihyperalgesic activity of a mexicanolide isolated from Swietenia humilis extract in nicotinamide-streptozotocin hyperglycemic mice. Biomed. Pharmacother. 2017, 92, 324-330. [CrossRef] [PubMed]

77. Maiti, A.; Dewanjee, S.; Kundu, M.; Mandal, S.C. Evaluation of antidiabetic activity of the seeds of Swietenia macrophylla in diabetic rats. Pharm. Biol. 2009, 47, 132-136. [CrossRef]

78. Zhang, B.; Wang, Z.F.; Tang, M.Z.; Shi, Y.L. Growth inhibition and apoptosis-induced effect on human cancer cells of toosendanin, a triterpenoid derivative from Chinese traditional medicine. Investig. New Drugs 2005, 23, 547-553. [CrossRef] [PubMed]

79. Akihisa, T.; Pan, X.; Nakamura, Y.; Kikuchi, T.; Takahashi, N.; Matsumoto, M.; Ogihara, E.; Fukatsu, M.; Koike, K.; Tokuda, H. Limonoids from the fruits of Melia azedarach and their cytotoxic activities. Phytochemistry 2013, 89, 59-70. [CrossRef] [PubMed]

80. Awang, K.; Chong, S.L.; Mohamad, K.; Morita, H.; Hirasawa, Y.; Takeya, K.; Thoison, O.; Hadi, A.H.A. Erythrocarpines A-E, new cytotoxic limonoids from Chisocheton erythrocarpus. Bioorg. Med. Chem. 2007, 15, 5997-6002. [CrossRef] [PubMed]

(C) 2018 by the authors. Licensee MDPI, Basel, Switzerland. This article is an open access article distributed under the terms and conditions of the Creative Commons Attribution (CC BY) license (http://creativecommons.org/licenses/by/4.0/). 